\title{
Stability Analysis of a Feedback Model for the Action of the Immune System in Leukemia
}

\author{
S. Balea ${ }^{1}$, A. Halanay ${ }^{1}$, D. Jardan ${ }^{1}$, M. Neamţu ${ }^{1,2}{ }^{*}$, C. A. Safta ${ }^{1}$ \\ 1 "POLITEHNICA" University of Bucharest Department of Mathematics and Informatics \\ Splaiul Independentei 313 RO-060042 Bucharest, Romania \\ ${ }^{2}$ West University of Timisoara, Department of Economics and Modelling \\ 300115 Pestalozzi Str. 16, Timisoara, Romania
}

\begin{abstract}
A mathematical model, coupling the dynamics of short-term stem-like cells and mature leukocytes in leukemia with that of the immune system, is investigated. The model is described by a system of seven delay differential equations with seven delays. Three equilibrium points $E_{0}, E_{1}, E_{2}$ are highlighted. The stability and the existence of the Hopf bifurcation for the equilibrium points are investigated. In the analysis of the model, the rate of asymmetric division and the rate of symmetric division are very important.
\end{abstract}

Keywords and phrases: immune system, leukemia, delay differential equations, stability, Hopf bifurcation, limit cycle

Mathematics Subject Classification: 34K20, 37G15, 92D25

\section{Introduction}

Mathematical modelling of biological, physical or social sciences phenomena need often the consideration of time delays through delay differential equations. Some models use discrete delays other distributed or continuous delays.

For the description of biological processes implied in hematopoiesis, a mathematical model that includes time delays will be used. It is based on the mass action principle, in the spirit of [4], [5], [6], [9], [15], [19] and [25]. The aim is the study of the dynamics of Chronic Myelogenous Leukemia (CML) when the action of different cell lines of the immune system is considered. Other authors ([17], [18]) used the more simple model from [21] for the dynamics of hematopoietic stem cells (HSC). Other approaches can be found in [3], [14], [24] [27].

The lethality of malignant tumors is determined in large part by their unregulated proliferative activity, the resistance of tumor cells to apoptotic death and the ability of tumor cells to invade host tissues and metastasize to distant sites. CML, also known as Chronic Granulocytic Leukemia, is a cancer of white blood cells. It is a clonal marrow stem cells disorder in which the main characteristic is the proliferation of granulocytes (neutrophils, eosinophils and basophils) and of their precursors in the bone marrow and

${ }^{*}$ Corresponding author. E-mail: mihaela.neamtu@feaa.uvt.ro 
the accumulation of these cells in the blood. It is a type of myeloproliferative disease associated to a chromosomal translocation called the Philadelphia chromosome (see also [10], [22]).

The possibility that cancers can be eradicated by specific immune responses has been the impetus for a large body of work in the field of tumor immunology (see [17], [18], [21], [23]). Several characteristics of tumor antigens and immune responses to tumors are fundamental to an understanding of tumor immunity and for the development of strategies for cancer immunotherapy. Histopathological studies show that many tumors are surrounded by mono-nuclear cells infiltrates composed of T lymphocytes, natural killer (NK) cells and macrophages. The immune surveillance and tumor immunity varies with the type of tumor.

The main sources for the presentation of the action of the immune system are [1] and [29].

Cell-mediated immune response plays a critical role in combating viral infection. As a virus infection becomes established in the host, a series of molecular and cellular signals are initiated, that activate cell-mediated immune responses. These signals include the production of interferons, other cytokines and inflammatory mediators that synergise the mobilization of local Antigen-Presenting Cells (APCs). The adaptive immune response is initiated when naive $\mathrm{T}$ cells encounter specific antigens on the surface of an APC.

The APCs such as Dendritic cells, Macrophages and B-lymphocytes are specialized blood cells that help to fight off foreign substances that enter the body.APCs are present in lymphoid organs, in the epithelia of the skin and gastrointestinal and respiratory tracts, and in the interstitium of most parenchymal organs. Resting (immature) APCs, usualy located in the epithelia, express membrane receptors that can capture and endocytose antigens which are then processed into peptides that bind to the major histocompatibility complex (MHC). The immature APC have low levels of MHC molecules on their surface and high capacity of fagocytosis. Immature APCs can also ingest antigens by micropinocytosis and macropinocytosis. APCs are activated by signals and cytokines (e.g TNF), produced during the innate immune response phase in response to the presence of antigens. The activated APCs (also called mature APCs) lose their adhesiveness for epithelia or tissues and migrate into lymph nodes. Mature APCs express high levels of MHC molecules with bound peptides as well as costimulator molecules required for $\mathrm{T}$ cell activation. Thus activation converts the APCs from fagocytes into cells that are able to present antigens and activate naive $\mathrm{T}$ cells.

Dendritic cells (DC) are the members of the immune system that have as their main function to process antigen material and present it on the surface to other cells of the immune system. Therefore, they function as antigen-presenting cells. They act as messengers between the innate and adaptive immunity. DC are present in tissues in contact with the external environment such as skin and the inner lining of nose, lungs, stomach and intestines. They can also be found, in an immature state, in blood.

The macrophages are white blood cells located within tissues, produced by the division of the monocytes and acting in both non-specific defence (innate immunity) and in the initiation of specific defence mechanisms (adaptive immunity).

The APCs are thought to provide a critical cellular link for naive (CD4+ and CD8+) T cells. Naive $\mathrm{T}$ cells fall into two large classes, one of which carries the co-receptor CD8+ on its surface and the other bears the co-receptor CD4+.

CD8 + $\mathrm{T}$ cells differentiate into CD8 + cytotoxic $\mathrm{T}$ cells that kill their target cells by inducing them to undergo apoptosis. They are important in the defense against intracellular pathogens, especially viruses. CD8 $+\mathrm{T}$ cells are potent antiviral effector cells due to their ability to produce both inflammatory mediators and cytotoxic effector molecules. CD8 $+\mathrm{T}$ cells are commonly referred to as cytotoxic $\mathrm{T}$ lymphocytes (CTLs), which emphasizes their ability to kill virally infected target cells. These killing functions are triggered as the effector $\mathrm{T}$ cells become activated.

Because the effector actions of these cells are so destructive, naive CD8+ T cells require more costimulatory activity to drive them to become activated effector cells than do naive CD4+ T cells.

CD8+ T cell activation requires additional help, which is provided by CD4+ effector T cells. Effector $\mathrm{CD} 4+\mathrm{T}$ cells that recognize related antigens presented by APCs can amplify the activation of CD8+ T 
cells: the cytokine IL-2, produced by effector CD4+ T cells, acts as a growth factor to promote CD8+ T cells differentiation. CD4+ T cells produce an array of cytokines and chemokines that stimulate cells of the innate immune system.

In contrast with CD8 $+\mathrm{T}$ cells, CD4 $\mathrm{T}$ cells differentiate into several subsets of effector $\mathrm{T}$ helper cells with a variety of different functions, the main functional classes are Th 1, Th 2, Th 17 and the regulatory cells. Th1 cells help control bacteria, Th 2 cells help to control infection by parasites. The main function of Th 17 cells seems to be to help protect against extracellular bacteria and fungi through stimulating the neutrophil response that helps to clear such pathogens. The regulatory CD4+ T cells are involved in controlling adaptive immune responses: in addition to their ability to prevent autoimmune disease, Treg cells have been shown to suppress antigen - specific $\mathrm{T}$ cells proliferation.

Although T-cells responses can be highly effective at controlling acute infections and contribute to protective secondary responses, persistent infections do arise and are often associated with the development of phenotypically and functionally inferior responses. The same situation is encountered in cancer as well. The immune cells respond to cancer cells in the same way they respond to infected cells. A common feature is that T-cells response is initially induced, but qualitative and quantitative defects become apparent as the generation of robust sets of effector cells, as well as the progression of memory T-cells'development, are subverted (see [1], [29]). The production of cytokines, including IL-2, TNF-a and IFN-g, as well as cytotoxic effector molecules such as perforin, may be diminished or abolished and decreased proliferative potential has also been observed. The severe loss of effector activity as well as the physical deletion of $\mathrm{T}$ cells that occur during cancers has been termed exhaustion.

The scheme of the interactions is:

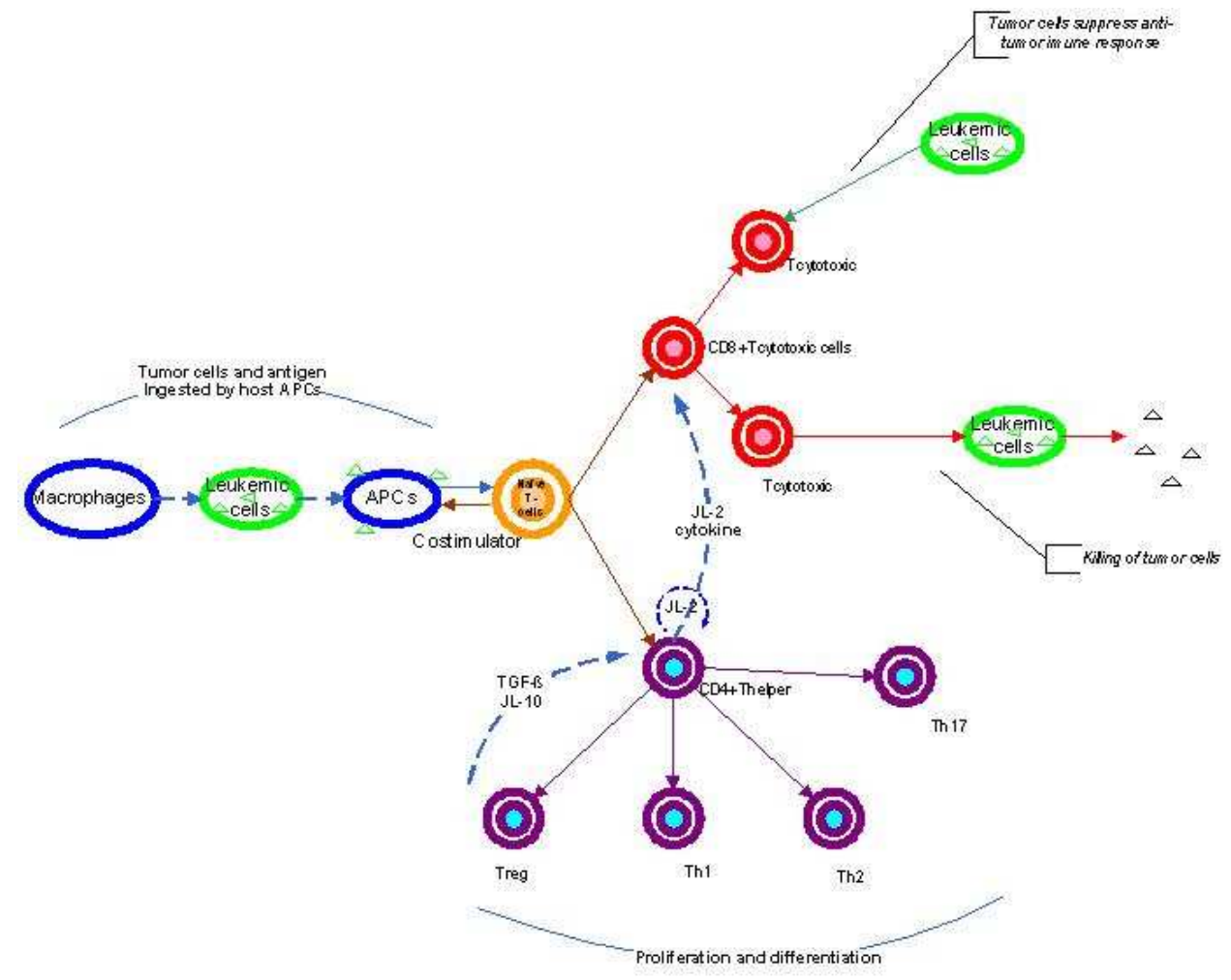

This paper studies a model that describes the interaction between leukemic cells and the immune system. After the description of the model, the local asymptotic stability of some equilibrium points is analyzed. We find sufficient conditions for the existence of Hopf bifurcation with respect to the delay $\tau_{1}$ considered as parameter. The paper is organized as follows. In Section 2 the description of the model is given. In Section 3 the equilibrium points $E_{0}, E_{1}, E_{2}$ are determined. In Section 4 the stability and the 
existence of the Hopf bifurcation for the equilibrium point $E_{0}$ are investigated. Section 5 deals with the stability of the equilibrium points $E_{1}$ and $E_{2}$. Numerical simulations are given in Section 6 . Section 7 is dedicated to concluding remarks.

\section{Description of the model}

In the present paper the property of asymmetric division, when from a stem cell results after mitosis a stem cell and a comitted cell, is taken into consideration in the model (see also [6], [20], [25], [26], [28]). Incorporating the antileukemia immune response, our model considers that anti-leukemia $\mathrm{T}$ cells (involving both $\mathrm{CD} 4+$ and $\mathrm{CD} 8+\mathrm{T}$ cells) response may play an important role in the dynamics of CML, for a while (see also [17], [18], [21], [22], [23]).

The hematopoietic stem cells that are considered are supposed to be in the proliferative phase or to spend a short time into the resting phase. These cells are called, following [20], Short-Term Hematopoietic Stem Cells (ST-HSC). In what follows $x_{1}$ denotes the density of short-term stem-like leukemia cells, $x_{2}$ the density of leukemia neutrophil cells, $x_{3}$ the concentration of immature APCs, $x_{4}$ the concentration of mature APCs, $x_{5}$ the concentration of passive $T$ cells of both CD4+ and CD $8+$ phenotype, $x_{6}$ the concentration of active CD4+T-helper cells and $x_{7}$ the concentration of active CD8+ cytotoxic T-cells. The following system of delay differential equations models the complex interactions between the cells of the immune system and between these ones and the leukemic cells:

$$
\begin{aligned}
& \dot{x}_{1}(t)=-a_{5} x_{1}(t)-\left(1-b_{1}-b_{2}\right) a_{3} \beta\left(x_{1}(t)\right) x_{1}(t)+2 e^{-b_{3} \tau_{1}}\left(1-b_{1}-b_{2}\right) a_{3} \beta\left(x_{1}\left(t-\tau_{1}\right)\right) x_{1}\left(t-\tau_{1}\right)- \\
& -\left(b_{1}+b_{2}\right) a_{4} k\left(x_{2}(t)\right) x_{1}(t)+e^{-b_{3} \tau_{1}} b_{1} a_{4} k\left(x_{2}\left(t-\tau_{1}\right)\right) x_{1}\left(t-\tau_{1}\right)-b_{4} \frac{x_{1}(t) x_{7}(t)}{1+b_{5}\left(x_{1}(t)+x_{2}(t)\right)} \\
& \dot{x}_{2}(t)=-b_{6} x_{2}(t)+b_{7} a_{4} k\left(x_{2}\left(t-\tau_{2}\right)\right) x_{1}\left(t-\tau_{2}\right)-b_{8} \frac{x_{2}(t) x_{7}(t)}{1+b_{5}\left(x_{1}(t)+x_{2}(t)\right)} \\
& \dot{x}_{3}(t)=-c_{2} x_{3}(t)+c_{1}-\frac{c_{3} x_{3}(t)\left(x_{1}(t)+x_{2}(t)\right)}{1+\left(x_{1}(t)+x_{2}(t)\right)^{p}} \\
& \dot{x}_{4}(t)=-d_{2} x_{4}(t)+\frac{c_{3} x_{3}(t)\left(x_{1}(t)+x_{2}(t)\right)}{1+\left(x_{1}(t)+x_{2}(t)\right)^{p}} \\
& \dot{x}_{5}(t)=-d_{4} x_{5}(t)+d_{3}-d_{5} x_{4}(t) x_{5}(t) \\
& \dot{x}_{6}(t)=-e_{1} x_{6}(t)-e_{2} \zeta_{1}\left(x_{6}(t)\right) x_{6}(t)+2 e^{-e_{1} \tau_{3}} e_{2} \zeta_{1}\left(x_{6}\left(t-\tau_{3}\right)\right) x_{6}\left(t-\tau_{3}\right)- \\
& -e_{3} \zeta_{2}\left(x_{6}(t)\right) x_{6}(t)+2^{m_{1}} d_{61} x_{4}\left(t-\tau_{5}\right) x_{5}\left(t-\tau_{5}\right) \\
& \left.\dot{x}_{7}(t)=-\left(1+e_{4}\right) x_{7}(t)-e_{7} \zeta_{3}\left(x_{6}(t)\right) x_{7}(t)+2 e^{-e_{4} \tau_{4}} e_{7} \zeta_{3}\left(x_{6}\left(t-\tau_{4}\right)\right) x_{7}\left(t-\tau_{4}\right)\right)- \\
& -b_{9} \frac{x_{7}(t)\left(x_{1}(t)+x_{2}(t)\right)}{1+e_{8}\left(x_{1}(t)+x_{2}(t)\right)}+2^{m_{1}} e_{9} \frac{x_{7}\left(t-n_{1} \tau_{4}\right)\left(x_{1}\left(t-n_{1} \tau_{4}\right)+x_{2}\left(t-n_{1} \tau_{4}\right)\right)}{1+e_{8}\left(x_{1}\left(t-n_{1} \tau_{4}\right)+x_{2}\left(t-n_{1} \tau_{4}\right)\right)}+ \\
& +2^{m_{2}} d_{62} x_{4}\left(t-\tau_{6}\right) x_{5}\left(t-\tau_{6}\right) .
\end{aligned}
$$

Here $p>1$ and the functions $\zeta_{1}, \zeta_{2}, \zeta_{3}, \beta, k$ are defined by:

$$
\begin{aligned}
& \zeta_{1}(u)=\frac{1}{1+u^{p_{2}}}, \quad \zeta_{2}(u)=\frac{u^{2}+e_{5}}{u^{2}+e_{6}}, \quad \zeta_{3}(u)=\frac{1}{1+u^{p_{3}}}, p_{2} \geq 2, p_{3} \geq 2, \\
& \beta(u)=\frac{a_{1}^{2 m}}{a_{1}^{2 m}+u^{2 m}}, m>1, \quad k(u)=\frac{a_{2}^{2 n}}{a_{2}^{2 n}+u^{2 n}}, n>1 .
\end{aligned}
$$

Our system of seven delay differential equations (DDEs) is considerably different from the systems used in references [17] and [18]. In the DDEs system in [18], the $T$ cell dynamics is considered in the absence of the leukemic cells which is not the case in our model. In [18] the state variables are $H_{0}$, 
the concentration of naive CD4+T cells, $H$ the concentration of effector CD4+T helper cells, $K_{0}$, the concentration of naive CD8+T cells, $K$, the concentration of effector CD8+ T cytotoxic cells, and $R$, the concentration of Tregs, and, in addition, $P$, the concentration of positive growth signal (e.g., IL-2).

In our model, instead of $H_{0}$ and $K_{0}$ only one variable, $x_{5}$, the concentration of passive $\mathrm{T}$ cells of both CD4+ and CD8+ phenotype is considered. Due to the fact that besides the regulatory $\mathrm{T}$ cells, there are other cell types involved in the regulatory mechanism, we do not consider an equation for Treg as in the reference [18], nor an equation for the concentration $P$, of positive growth signal as in [18], keeping in mind that active CD4+ T helper cells secrete positive growth signals at different rates, but instead we introduce three feedback, T helper depending, functions, $\zeta_{1}\left(x_{6}(t)\right), \zeta_{2}\left(x_{6}(t)\right)$ and $\zeta_{3}\left(x_{6}(t)\right)$. Indeed, knowing that active CD4+ T helper cells secrete positive growth signals (e.g. cytokines, IL- 2), at different rates, for self development, (the autocrine loop), and to stimulate T cytotoxic cells for further division, on one side, and on the other side, that there are suppressive signals against $\mathrm{T}$ helper and $\mathrm{T}$ cytotoxic cells, due to the regulatory process, (depending on the $\mathrm{T}$ helper concentration), we are led to consider the functions $\zeta_{1}, \zeta_{2}, \zeta_{3}$ in the 6 th and 7 th equations.

The initial conditions of system $(2.1)$ will be defined on the interval $\left[-\tau_{m}, 0\right]$ where $\tau_{m}=$ $\max \left\{\tau_{1}, \tau_{2}, \tau_{3}, \tau_{4}, \tau_{5}, \tau_{6}, n_{1} \tau_{4}\right\}\left(x_{i}(\theta)=x_{i}(0), i=1 . .7, \theta \in\left[-\tau_{m}, 0\right]\right)$

The coefficients of system (2.1) are positive real numbers and their interpretations are given in the table from the numerical simulation.

The delays in system $(2.1)$ are $\tau_{1}, \tau_{2}, \tau_{3}, \tau_{4}, \tau_{5}, \tau_{6}$ and $n_{1} \tau_{4}$.

The first two equations, for the density of stem-like leukemia cells and of the leukemia neutrophil cells, are similar to those in [25] (see also [10], [15]).

The scheme of the first two equations in $(2.1)$ is:

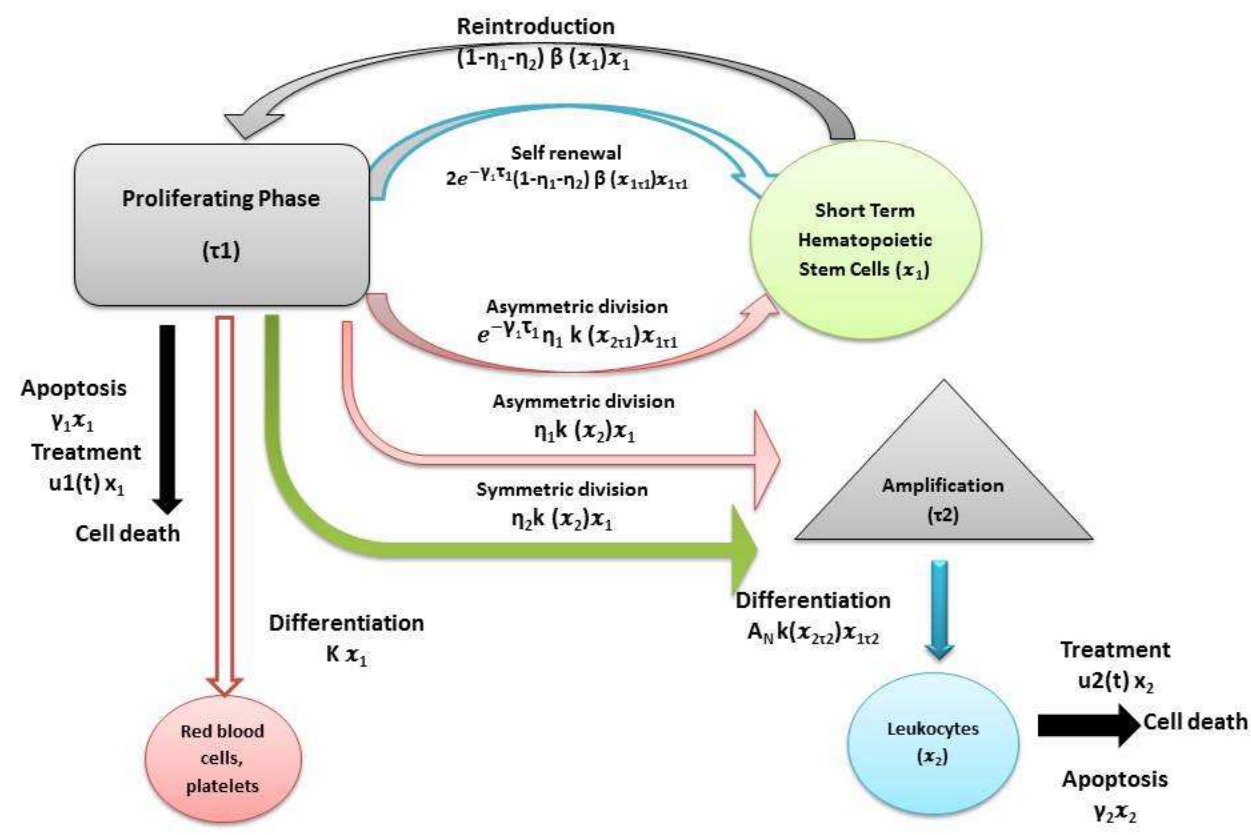

In our model, the last terms in the first two equations model the influence of cytotoxic $\mathrm{T}$ cells on the disease.

The time necessary for a ST-HSC to complete a cycle of self-renewal, asymmetric division or differentiation is supposed to be the same, $\tau_{1}$. The time necessary for the maturation of leukocytes is denoted by $\tau_{2}$. 
The equations for $x_{3}$ (naive APCs), $x_{4}$ ( active APCs ) and $x_{5}$ (inactive T cells) are similar to those in [18], but with the significant difference that the rate of antigen stimulation is not anymore the function $\mathrm{a}(\mathrm{t})$ as in [18], but a feedback function, $-\frac{c_{3} x_{3}\left(x_{1}+x_{2}\right)}{1+\left(x_{1}+x_{2}\right)^{p}}$, depending on the level of leukemic cells. Also, memory $\mathrm{T}$ cells are regarded in our presentation as a part of inactive $\mathrm{T}$ cells (independent on their appartenance to CD4/CD8 phenotype).

In the 6-th equation the last term gives the rate at which naive CD4+ $\mathrm{T}$ cells enter the active state after finishing the minimal developmental program of $m_{1}$ cell divisions and the time delay $\tau_{5}$ is the duration of the minimal developmental program. The first term indicates that CD4+ T helper cells exit the population through death at the rate $e_{1}$. Active CD4+ T helper cells secrete positive growth signal at different rates: CD4+ cells provides signals, cytokines, IL- 2, for self development, the autocrine loop described by the function $\zeta_{1}(t)$. The second term in the equation of $x_{6}(t)$ is the rate at which $\mathrm{T}$ helper cells are stimulated by themselves for further division, and the third term is the rate in which cells reenter the effector CD4+ population after having divided once. The time delay $\tau_{3}$ is the duration of one CD4+ cell division.

Because, besides the regulatory $\mathrm{T}$ cells, there are other cell types involved in the regulatory mechanism, we will not consider an equation for Treg and the regulatory process will be expressed through a $\mathrm{T}$ helper $\left(x_{6}\right)$ dependent function, $\zeta_{2}\left(x_{6}(t)\right)$. This is another difference to the Kim et al. model. The fourth term is the rate at which $\mathrm{T}$ helper cells are suppressed by regulatory activity of the immune system.

In the last equation of the system the variation of CTLs concentration is described. The last term gives the rate at which activated CD8 $+\mathrm{T}$ cells enter the effector state after finishing the minimal developmental program of $m_{2}$ cell divisions and the delay $\tau_{6}$ is the duration of this minimal developmental program. The first term is the rate at which CD8+ cytotoxic $\mathrm{T}$ cells exit the population through death at rate $(1+\mathrm{e} 4)$. The parameter $(1+\mathrm{e} 4)$ is composed by both the apoptosis rate, and the rate due to regulatory mechanisms. The second term is the rate at which $\mathrm{T}$ cytotoxic cells are stimulated by positive growth signal (Il-2) secreted by CD4+T helper cells, for further division, on one side, and on the other side, are suppressed by Tregs. The third term is the rate at which cells reenter the effector cytotoxic population after having divided once. The time delay $\tau_{4}$ is the duration of one cycle of CD $8+$ cell division.

Leukemia cells suppress anti-leukemia immune response. The precise mechanism is unknown. It is assumed that the level of down-regulation depends on the current leukemia population (see [18]) and this suppresive action is expressed by the presence of the entire population of leukemic cells $\left(x_{1}\right.$ and $\left.x_{2}\right)$ in the denominator of the fourth and the fifth terms.

System (2.1) contains seven delays $\tau_{i}, i=1 . .7$, where $\tau_{7}=n_{1} \tau_{4}$, with $n_{1}$ the number of antigen dependent divisions. In the right-hand side of the system the following delay-dependent terms are present: $e^{-b_{3} \tau_{1}}, e^{-e_{1} \tau_{3}}, e^{-e_{4} \tau_{4}}$. Thus (2.1) is a delay differential system with delay-dependent parameters. For the analysis of this system we use [8], [9], [11], [12], [13].

Define $\tau=\max \left\{\tau_{i}, i=1 \ldots 7\right\}$ and consider the initial data $\varphi$ in $C\left([-\tau, 0], \mathbb{R}^{7}\right)$. Since the right-hand side functions $f_{i}, \mathrm{i}=1 \ldots 7$, are $C^{1}$, local solutions will exist whenever the initial data comply with the domain of existence of each of these functions and in particular when these initial conditions are positive.

It is not difficult to see that if the initial conditions have all components positive functions, the solutions of the system will have positive components on all the interval of existence. Indeed, if $x_{1}(\theta)>0$ for any $\theta \in[-\tau, 0]$ and there exists $T>0$ such that $x_{1}(T)=0$ the derivative $\dot{x}_{1}(T)$ will be positive and this leads to a contradiction. The same argument works for the other components, too. 


\section{Determination of some equilibrium points}

The equilibrium points are given by the solutions of the following system:

$$
\begin{aligned}
& x_{1}\left(a_{5}+\left(1-2 e^{-b_{3} \tau_{1}}\right)\left(1-b_{1}-b_{2}\right) a_{3} \beta\left(x_{1}\right)+a_{4}\left(b_{1}+b_{2}-b_{1} e^{-b_{3} \tau_{1}}\right) k\left(x_{2}\right)+\right. \\
& \left.+b_{4} \frac{x_{7}}{1+b_{5}\left(x_{1}+x_{2}\right)}\right)=0 \\
& x_{2}\left(b_{6}+b_{8} \frac{x_{7}}{1+b_{5}\left(x_{1}+x_{2}\right)}\right)-b_{7} a_{4} k\left(x_{2}\right) x_{1}=0 \\
& x_{3}\left(c_{2}+\frac{x_{1}+x_{2}}{1+\left(x_{1}+x_{2}\right)^{p}}\right)-c_{1}=0 \\
& d_{2} x_{4}-c_{3} \frac{x_{3}\left(x_{1}+x_{2}\right)}{1+\left(x_{1}+x_{2}\right)^{p}}=0 \\
& x_{5}\left(d_{4}+d_{5} x_{4}\right)-d_{3}=0 \\
& x_{6}\left(e_{1}+e_{2} \zeta_{1}\left(x_{6}\right)\left(1-2 e^{-e_{1} \tau_{3}}\right)+e_{3} \zeta_{2}\left(x_{6}\right)\right)-2^{m_{1}} d_{61} x_{4} x_{5}=0 \\
& x_{7}\left(1+e_{4}+e_{7}\left(1-2 e^{-e_{4} \tau_{4}}\right) \zeta_{3}\left(x_{6}\right)+\left(b_{9}-2^{m_{1}} e_{9}\right) \frac{x_{1}+x_{2}}{1+e_{8}\left(x_{1}+x_{2}\right)}\right)=0
\end{aligned}
$$

Proposition 1. The system (3.1) has always the solution (equilibrium point for (2.1)) $E_{0}$ with the coordinates:

$$
x_{10}=0, x_{20}=0, x_{30}=\frac{c_{1}}{c_{2}}, x_{40}=0, x_{50}=\frac{d_{3}}{d_{4}}, x_{60}=0, x_{70}=0 .
$$

The proof is obvious.

Proposition 2. If $\tau_{1} \geq 0, \tau_{4} \geq \frac{\ln 2}{e_{4}}, e_{2}>e_{1}, e_{6}>e_{5}$ and $T_{30} \leq \tau_{3}<T_{31}$, where

$$
T_{30}=\frac{\ln \left(2 e_{2} /\left(e_{1}+e_{2}+e_{3}\right)\right)}{e_{1}}, T_{31}=\frac{1}{e_{1}} \ln \frac{2 e_{2} e_{6}}{e_{1} e_{6}+e_{2} e_{6}+e_{3} e_{5}}
$$

then there exists a unique equilibrium point $E_{1}$ with the coordinates:

$$
x_{11}=0, x_{21}=0, x_{31}=\frac{c_{1}}{c_{2}}, x_{41}=0, x_{51}=\frac{d_{3}}{d_{4}}, x_{61}=x_{6}^{*}, x_{71}=0,
$$

where $x_{6}^{*}$ is the positive root of the equation:

$$
\begin{aligned}
& F\left(x_{6}\right):=\left(e_{1}+e_{3}\right) x_{6}^{p_{2}+2}+\left(e_{1} e_{6}+e_{3} e_{5}\right) x_{6}^{p_{2}}+\left(e_{1}+e_{3}+e_{2}\left(1-2 e^{-e_{1} \tau_{3}}\right)\right) x_{6}^{2}+ \\
& +e_{1} e_{6}+e_{3} e_{5}+e_{2} e_{6}\left(1-2 e^{-e_{1} \tau_{3}}\right)=0 .
\end{aligned}
$$

Proof. Remark first that all the coefficients in the first five equations of (3.1) are positive. So one obtains the first five components of $E_{1}$. We look for a non-zero sixth component so, from the sixth equation of (3.1), we have:

$$
e_{1}+e_{2}\left(1-2 e^{-e_{1} \tau_{3}}\right) \frac{1}{1+x_{6}^{p_{2}}}+e_{3} \frac{x_{6}^{2}+e_{5}}{x_{6}^{2}+e_{6}}=0 .
$$

From (3.6) we obtain (3.5). Deriving $F\left(x_{6}\right)$ with respect to $x_{6}$ we have:

$$
F^{\prime}\left(x_{6}\right)=\left(p_{2}+2\right)\left(e_{1}+e_{3}\right) x_{6}^{p_{2}+1}+p_{2}\left(e_{1} e_{6}+e_{3} e_{5}\right) x_{6}^{p_{2}-1}+\left(e_{1}+e_{3}+e_{2}\left(1-2 e^{-e_{1} \tau_{3}}\right)\right) x_{6} .
$$

Because $\tau_{3}>T_{30}$, then the coefficients of $F^{\prime}\left(x_{6}\right)$ are positive. Thus, $F^{\prime}\left(x_{6}\right)>0$, for $x_{6} \geq 0$. Then, $F\left(x_{6}\right)$ is a strictly increasing function for $x_{6} \geq 0$. We have: 


$$
F(0)=e_{1} e_{2}+e_{3} e_{5}+e_{2} e_{6}\left(1-2 e^{-e_{1} \tau_{3}}\right) .
$$

Therefore, if the inequality

$$
e_{1} e_{6}+e_{3} e_{5}+e_{2} e_{6}\left(1-2^{-e_{1} \tau_{3}}\right)<0
$$

holds true, then Eq. (3.5) has a unique positive root denoted by $x_{6}^{*}$. From (3.7) we obtain $T_{31}$. Remark that $T_{30}<\tau_{3}<T_{31}<\frac{\ln 2}{e_{1}}$.

The proposition is proved.

Proposition 3. If $\tau_{1} \geq 0, e_{2}>e_{1}, e_{6}>e_{5}, T_{30} \leq \tau_{3}<T_{31}$ and $e_{7}>\left(1+e_{4}\right)\left(1+x_{6}^{* p_{3}}\right)$ then, if $\tau_{4}=T_{40}$, with

$$
T_{40}=\frac{1}{e_{4}} \ln \frac{2 e_{7}}{\left(1+e_{4}\right)\left(1+x_{6}^{* p_{3}}\right)+e_{7}}
$$

where $x_{6}^{*}$ is the positive root of Eq. (3.5), there exist equilibrium points $E_{2}$ with the coordinates:

$$
x_{12}=0, x_{22}=0, x_{32}=\frac{c_{1}}{c_{2}}, x_{42}=0, x_{52}=\frac{d_{3}}{d_{4}}, x_{62}=x_{6}^{*}, x_{72}=c
$$

$c \geq 0$.

Proof. From the seventh equation of (3.1), we determine $0 \leq \tau_{4}$ so that

$$
1+e_{4}+e_{7}\left(1-2 e^{-e_{4} \tau_{4}}\right) \frac{1}{1+x_{6}^{* p_{3}}}=0 .
$$

When (3.10) is solved with respect to $\tau_{4}$ the value in (3.8) is obtained. Thus, there exist the equilibrium points $E_{2}$ with the coordinates given by (3.9).

Remark 4. The delay $\tau_{3}$ has a special role for existence of solutions of the system (2.1). If $\tau_{3} \geq \frac{\ln 2}{e_{1}}$ the only solution is $E_{0}$ and if $T_{30} \leq \tau_{3}<T_{31}$, then there exists also the solution $E_{1}$. In Proposition 3 , $\tau_{4}=T_{40}$ depends on $\tau_{3} \in\left[T_{30}, T_{31}\right)$ and the conditions above are only sufficient.

\section{Stability analysis for the equilibrium point $E_{0}$}

Consider the transformation: $u_{1}(t)=x_{1}(t), u_{2}(t)=x_{2}(t), u_{3}(t)=x_{3}(t)-c_{1} / c_{2}, u_{4}(t)=x_{4}(t)$, $u_{5}(t)=x_{5}(t)-d_{3} / d_{4}, u_{6}(t)=x_{6}(t), u_{7}(t)=x_{7}(t)$.

In order to analyze the stability of the equilibrium point $E_{0}$, we study the linearization in zero of system (2.1) obtained by this translation:

$$
\begin{aligned}
& \dot{u}_{1}(t)=a_{11} u_{1}(t)+b_{111} u_{1}\left(t-\tau_{1}\right), \\
& \dot{u}_{2}(t)=a_{22} u_{2}(t)+b_{212} u_{1}\left(t-\tau_{2}\right), \\
& \dot{u}_{3}(t)=a_{31} u_{1}(t)+a_{32} u_{2}(t)+a_{33} u_{3}(t), \\
& \dot{u}_{4}(t)=a_{41} u_{1}(t)+a_{42} u_{2}(t)+a_{44} u_{4}(t), \\
& \dot{u}_{5}(t)=a_{54} u_{4}(t)+a_{55} u_{5}(t), \\
& \dot{u}_{6}(t)=a_{66} u_{6}(t)+b_{663} u_{6}\left(t-\tau_{3}\right)+b_{645} u_{4}\left(t-\tau_{5}\right) \\
& \dot{u}_{7}(t)=a_{77} u_{7}(t)+b_{746} u_{4}\left(t-\tau_{6}\right)+b_{774} u_{7}\left(t-\tau_{4}\right)
\end{aligned}
$$

where 


$$
\begin{aligned}
& a_{11}=-a_{5}-\left(b_{1}+b_{2}\right) a_{4}-\left(1-b_{1}-b_{2}\right) a_{3}, b_{111}=e^{-b_{3} \tau_{1}}\left(2\left(1-b_{1}-b_{2}\right) a_{3}+a_{4} b_{1}\right), \\
& a_{22}=-b_{6}, b_{212}=-a_{4} b_{7}, a_{31}=-c_{1}, a_{32}=-c_{1}, a_{33}=-c_{2}, \\
& a_{41}=\frac{c_{1} c_{3}}{c_{2}}, a_{42}=\frac{c_{1} c_{3}}{c_{2}}, a_{44}=-d_{2}, \\
& a_{54}=-\frac{d_{5} d_{3}}{d_{4}}, a_{55}=-d_{4}, a_{66}=-e_{1}-e_{2}-\frac{e_{3} e_{5}}{e_{6}}, \\
& b_{663}=2 e_{2} e^{-e_{1} \tau_{3}}, b_{645}=\frac{2^{m_{1}} d_{61} d_{3}}{d_{4}} \\
& a_{77}=-1-e_{4}-e_{7}, \\
& b_{746}=\frac{2^{m_{1}} d_{62} d_{3}}{d_{4}}, b_{774}=2 e_{7} e^{-e_{4} \tau_{4}} .
\end{aligned}
$$

The characteristic equation of system (4.1) is:

$$
\begin{aligned}
& \left(\lambda-a_{11}-b_{111} e^{-\lambda \tau_{1}}\right)\left(\lambda-a_{22}\right)\left(\lambda-a_{33}\right)\left(\lambda-a_{44}\right)\left(\lambda-a_{55}\right) \\
& \cdot\left(\lambda-a_{66}-b_{663} e^{-\lambda \tau_{3}}\right)\left(\lambda-a_{77}-b_{774} e^{-\lambda \tau_{4}}\right)=0 .
\end{aligned}
$$

The roots of (4.3) are given by:

$$
\lambda_{2}=a_{22}=-b_{6}, \lambda_{3}=a_{33}=-c_{3}, \lambda_{4}=a_{44}=-d_{2}, \lambda_{5}=a_{55}=-d_{4},
$$

and by the solutions of the equations:

$$
\begin{aligned}
& \lambda-a_{11}-b_{111} e^{-\lambda \tau_{1}}=0, \\
& \lambda-a_{66}-b_{663} e^{-\lambda \tau_{3}}=0, \\
& \lambda-a_{77}-b_{774} e^{-\lambda \tau_{4}}=0,
\end{aligned}
$$

If $b_{6}>0, c_{3}>0, d_{2}>0, d_{4}>0$, then the roots in (4.4) are negative.

The equation given by (4.5) will be written as

$$
\lambda+\alpha_{1}+\beta_{1} e^{-b_{3} \tau_{1}} e^{-\lambda \tau_{1}}=0
$$

where $\alpha_{1}, \beta_{1}$ are given by:

$$
\begin{aligned}
& \alpha_{1}=a_{5}+\left(b_{1}+b_{2}\right) a_{4}+\left(1-b_{1}-b_{2}\right) a_{3}, \\
& \beta_{1}=-2\left(1-b_{1}-b_{2}\right) a_{3}-a_{4} b_{1} .
\end{aligned}
$$

If $b_{1}+b_{2}<1$ then $\alpha_{1}>0$ and $\beta_{1}<0$.

We will study the stability analyzing the roots of the equation (4.8) when all the parameters are fixed, except $\tau_{1}$, considered as variable.

Eq. (4.8) has one coefficient depending on $\tau_{1}$. For the analysis of the roots of Eq. (4.8) we apply the method from [8].

If $\tau_{1}=0$, equation (4.8) becomes:

$$
\lambda+\alpha_{1}+\beta_{1}=0 .
$$

Then (4.10) has the root $\lambda=-\alpha_{1}-\beta_{1}$. In what follows, we determine the value of $\tau_{1}$, denoted by $\tau_{1}^{0}$, for which in (4.8) eventually a change of stability might occur with the appearance of a Hopf bifurcation. 
Let $\lambda=i \omega\left(\tau_{1}\right)$ be a root of equation (4.8). It follows that:

$$
i \omega\left(\tau_{1}\right)+\alpha_{1}+\beta_{1} e^{-b_{3} \tau_{1}}\left(\cos \left(\omega\left(\tau_{1}\right) \tau_{1}\right)-i \sin \left(\omega\left(\tau_{1}\right) \tau_{1}\right)\right)=0
$$

Thus,

$$
\begin{aligned}
& \omega\left(\tau_{1}\right)=\beta_{1} e^{-b_{3} \tau_{1}} \sin \left(\omega\left(\tau_{1}\right) \tau_{1}\right), \\
& \alpha_{1}=-\beta_{1} e^{-b_{3} \tau_{1}} \cos \left(\omega\left(\tau_{1}\right) \tau_{1}\right) .
\end{aligned}
$$

From (4.11), we get:

$$
\omega^{2}\left(\tau_{1}\right)=\beta_{1}^{2} e^{-2 b_{3} \tau_{1}}-\alpha_{1}^{2} .
$$

Relation (4.12) implies that the condition $\omega^{2}\left(\tau_{1}\right)>0$ holds for $0<\tau_{1}<\tau_{1}^{1}$, where

$$
\tau_{1}^{1}=\frac{1}{b_{3}} \ln \left(\frac{\left|\beta_{1}\right|}{\alpha_{1}}\right)
$$

with $\left|\beta_{1}\right|>\alpha_{1}$.

The eigenvalues $\lambda_{+}=i \omega\left(\tau_{1}\right), \lambda_{-}=-i \omega\left(\tau_{1}\right), \omega\left(\tau_{1}\right)>0$ can only occur for $\tau_{1}$ in the interval $I=\left(0, \tau_{1}^{1}\right)$. There are no stability switches for $\tau_{1}>\tau_{1}^{1}$ since there are no pure imaginary roots of $(4.8)$.

From (4.11), let $\theta\left(\tau_{1}\right) \in[0,2 \pi)$ be defined by:

$$
\sin \theta\left(\tau_{1}\right)=\frac{\omega\left(\tau_{1}\right)}{\beta_{1} e^{-b_{3} \tau_{1}}}, \quad \cos \theta\left(\tau_{1}\right)=-\frac{\alpha_{1}}{\beta_{1} e^{-b_{3} \tau_{1}}} .
$$

Then a stability switch may occur, through the roots $\lambda= \pm i \omega\left(\tau_{1}\right)$, where $\omega\left(\tau_{1}\right)>0$ is given by (4.12) with the values $\tau_{1 n}$ given by

$$
\tau_{1 n}=\frac{\theta\left(\tau_{1}\right)+2 n \pi}{\omega\left(\tau_{1}\right)}, \quad \text { for } \text { some } n \in \mathbb{N}
$$

For each $n \in \mathbb{N}$ the mappings $\tau_{1 n}: I \rightarrow \mathbb{R}_{+}$are defined, where $I=\left(0, \tau_{1}^{1}\right)$, and the stability switch may occur only for the values of $\tau_{1}$ at which $\tau_{1 n}\left(\tau_{1}\right)=\tau_{1}$ for some $n \in N_{0}$. Hence (4.12) and (4.14) define the mapping (4.15) and the occurrence of stability switches takes place at the zeros of the function

$$
S_{n}\left(\tau_{1}\right)=\tau_{1}-\tau_{1 n}\left(\tau_{1}\right), \quad n \in \mathbb{N} .
$$

For $\tau_{1} \in I, \theta\left(\tau_{1}\right)$ is continuous and differentiable in $\tau_{1}$. In order to prove this, we notice that $\alpha_{1}+$ $\beta_{1} e^{-b_{3} \tau_{1}}<0$, for $\tau_{1}<\tau_{1}^{1}$ and $\cos \theta\left(\tau_{1}\right) \neq 1$ for $\tau_{1} \in I$. Hence for $\tau_{1} \in I, \theta\left(\tau_{1}\right) \in(0,2 \pi)$. Then, $\theta\left(\tau_{1}\right)$ and $\tau_{1 n}\left(\tau_{1}\right)$ are continuous and differentiable for $\tau_{1} \in I$.

We would like to see if it is possible to determine the direction in which the pair of imaginary roots $\lambda= \pm i \omega\left(\tau_{1}^{*}\right)$, where $\tau_{1}^{*}$ is such that $S_{n}\left(\tau_{1}^{*}\right)=0$ for some $n \in \mathbb{N}$, crosses the imaginary axis, when $\tau_{1}$ increases.

From Theorem 2.1 in [8] we have:

$$
\operatorname{sign}\left(\left.\frac{d \operatorname{Re}(\lambda)}{d \tau_{1}}\right|_{\lambda=i \omega\left(\tau_{1}^{*}\right)}\right)=\operatorname{sign}\left(\left.\frac{d S_{n}\left(\tau_{1}\right)}{d \tau_{1}}\right|_{\tau_{1}=\tau_{1}^{*}}\right)
$$

Hence, we have the following:

Proposition 5.( [8]) The equation (4.8) admits a pair of simple and conjugate roots $\lambda_{+}\left(\tau_{1}^{*}\right)=i \omega\left(\tau_{1}^{*}\right)$, $\lambda_{-}\left(\tau_{1}^{*}\right)=-i \omega\left(\tau_{1}^{*}\right), \omega\left(\tau_{1}^{*}\right)>0$ at $\tau_{1}^{*} \in I$ if $S_{n}\left(\tau_{1}^{*}\right)=0$, for some $n \in N_{0}$. The pair of simple conjugate pure imaginary roots crosses the imaginary axis from left to right if $\delta\left(\tau_{1}^{*}\right)>0$ and crosses the imaginary axis from right to left if $\delta\left(\tau_{1}^{*}\right)<0$, where

$$
\delta\left(\tau_{1}^{*}\right)=\operatorname{sign}\left(\left.\frac{d \operatorname{Re}(\lambda)}{d \tau_{1}}\right|_{\lambda=i \omega\left(\tau_{1}^{*}\right)}\right)
$$


The following analytical result on $R\left(\tau_{1}\right)$ is useful for determining the value of $\tau_{1}$ at which a stability switch occurs.

Proposition 6. For the equation (4.8) we have:

$$
R_{1}\left(\tau_{1}^{*}\right)=\operatorname{sign}\left\{\left.\frac{d \operatorname{Re}(\lambda)}{d \tau_{1}}\right|_{\lambda=i \omega\left(\tau_{1}^{*}\right)}\right\}=\operatorname{sign}\left\{\omega\left(\tau_{1}^{*}\right) \omega^{\prime}\left(\tau_{1}^{*}\right)\left(\alpha_{1}+\beta_{1} e^{-b_{3} \tau_{1}^{*}} \tau_{1}^{*}\right)+\omega^{2}\left(\tau_{1}^{*}\right) \beta_{1}^{2} e^{-2 b_{3} \tau_{1}^{*}}\right\}
$$

and

$$
R_{1}\left(\tau_{1}^{*}\right)=\operatorname{sign}\left\{-\alpha_{1}\left(b_{3}+\alpha_{1}\right)-\beta_{1}^{2}\left(b_{3} \tau_{1}^{*}-1\right) e^{-2 b_{3} \tau_{1}^{*}}\right\} .
$$

Proof. From (4.8) with $\lambda=\lambda\left(\tau_{1}\right)$ we obtain:

$$
\frac{d \lambda}{d \tau_{1}}=\frac{\lambda \beta_{1} e^{-b_{3} \tau_{1}}+\beta_{1} b_{3} e^{-b_{3} \tau_{1}}}{e^{\lambda \tau_{1}}-\beta_{1} e^{-b_{3} \tau_{1}} \tau_{1}}
$$

It is convenient to consider $\left(\frac{d \lambda}{d \tau_{1}}\right)^{-1}$. We have:

$$
\left(\frac{d \lambda}{d \tau_{1}}\right)^{-1}=\frac{e^{\lambda \tau_{1}}-\beta_{1} \tau_{1} e^{-b_{3} \tau_{1}}}{\lambda \beta_{1} e^{-b_{3} \tau_{1}}+b_{3} \beta_{1} e^{-b_{3} \tau_{1}}} .
$$

From (4.8) we obtain that:

$$
e^{\lambda \tau_{1}}=-\frac{\beta_{1} e^{-b_{3} \tau_{1}}}{\lambda+\alpha_{1}}
$$

Then,

$$
\left(\frac{d \lambda}{d \tau_{1}}\right)^{-1}=\frac{-\frac{1}{\lambda+\alpha_{1}}-\tau_{1}}{\lambda+b_{3}}
$$

Thus,

$$
\left.\left(\frac{d \lambda}{d \tau_{1}}\right)^{-1}\right|_{\lambda=i \omega\left(\tau_{1}\right)}=\frac{-\frac{1}{\omega^{2}\left(\tau_{1}\right)+\alpha_{1}^{2}}\left(\alpha_{1}-i \omega\left(\tau_{1}\right)\right)-\tau_{1}}{i \omega\left(\tau_{1}\right)+b_{3}} .
$$

Because $\omega^{2}\left(\tau_{1}\right)+\alpha_{1}^{2}=\beta_{1}^{2} e^{-2 b_{3} \tau_{1}}$ we obtain:

$$
\left.\left(\frac{d \lambda}{d \tau_{1}}\right)^{-1}\right|_{\lambda=i \omega\left(\tau_{1}\right)}=\frac{-\left(\alpha_{1}+\beta_{1}^{2} \tau_{1} e^{-2 b_{3} \tau_{1}}\right)+i \omega\left(\tau_{1}\right)}{\beta_{1}^{2} e^{-2 b_{3} \tau_{1}}\left(b_{3}+i \omega\left(\tau_{1}\right)\right)} .
$$

and

$$
\operatorname{Re}\left(\left.\left(\frac{d \lambda}{d \tau_{1}}\right)^{-1}\right|_{\lambda=i \omega\left(\tau_{1}\right)}\right)=\frac{\varphi_{1}\left(\tau_{1}\right)}{\beta_{1}^{2} e^{-2 b_{3} \tau_{1}}\left(b_{3}^{2}+\omega^{2}\left(\tau_{1}\right)\right)}
$$

where

$$
\varphi_{1}\left(\tau_{1}\right)=-\alpha_{1}\left(\alpha_{1}+b_{3}\right)+\beta_{1}^{2}\left(1-b_{3} \tau_{1}\right) e^{-2 b_{3} \tau_{1}}
$$

Therefore, we have that:

$$
R_{1}\left(\tau_{1}\right)=\operatorname{sign}\left(\varphi_{1}\left(\tau_{1}\right)\right)
$$

and that proves the proposition.

Proposition 6 can be completed with the study of the variation of $\varphi_{1}$ :

Proposition 7. Assume that

$$
\varphi_{1}(0) \varphi_{1}\left(\tau_{1}^{1}\right)<0 .
$$

Then, there exists the value $\tau_{1}^{0}, 0<\tau_{1}^{0}<\tau_{1}^{1}$ so that

$$
\operatorname{sign}\left\{\left.\frac{d \operatorname{Re}(\lambda)}{d \tau_{1}}\right|_{\lambda=i \omega\left(\tau_{1}\right)}\right\}
$$


changes signs in $\left(0, \tau_{1}^{0}\right)$ and in $\left(\tau_{1}^{0}, \tau_{1}^{1}\right)$.

The value $\tau_{1}^{0}$ is the unique zero of the function

$$
\varphi_{1}\left(\tau_{1}\right)=\left(1-b_{3} \tau_{1}\right) \beta_{1}^{2} e^{-2 b_{3} \tau_{1}}-\alpha_{1}\left(b_{3}+\alpha_{1}\right), \quad \tau_{1} \in\left(0, \tau_{1}^{1}\right) .
$$

Remark 5. Condition (4.21) is fulfiled whenever

$$
\beta_{1}^{2}>\alpha_{1}\left(\alpha_{1}+b_{3}\right)
$$

Indeed, $\varphi_{1}(0)=\beta_{1}^{2}-\alpha_{1}\left(\alpha_{1}+b_{3}\right)$ will be positive and $\varphi_{1}\left(\tau_{1}^{1}\right)=-\alpha_{1}\left(b_{3}+\alpha_{1} \ln \left(\left|\beta_{1}\right| / \alpha_{1}\right)\right)$ will be negative.

In the next proposition, we summarize the properties of the function $S_{n}\left(\tau_{1}\right)$.

Proposition 8. Assume that the function $S_{n}\left(\tau_{1}\right)$ has a positive root $\tau_{1}^{*} \in I$ for some $n \in N_{0}$, then a pair of simple purely imaginary roots $\pm i \omega\left(\tau_{1}^{*}\right)$ of equation (4.8) exists at $\tau_{1}=\tau_{1}^{*}$. In addition:

(i) If $S_{n}\left(\tau_{1}^{*}\right)=0$, then this pair of simple conjugate pure imaginary roots crosses the imaginary axis from left to right if $\delta\left(\tau_{1}^{*}\right)>0$ and from right to left if $\delta\left(\tau_{1}^{*}\right)<0$, where $\delta\left(\tau_{1}^{*}\right)$ is given by (4.18).

(ii) If $S_{0}\left(\tau_{1}\right)$ has no zero on $I$, the function $S_{n}\left(\tau_{1}\right)$ has no zero on I for all $n \geq 1$.

(iii) If for some $n \in N_{0}$ the functions $S_{n}\left(\tau_{1}\right)$ become zero for some time delay lags $\tau_{1 n} \in I$, then there exits at least one $\tau_{1 n_{j}}$ satisfying $\frac{d S_{n}\left(\tau_{1 n_{j}}\right)}{d \tau_{1}} \neq 0$. Define

$$
\left\{\tau_{1 j} \mid \tau_{1 j}<\tau_{1 j+1}, \quad j=0,1, \ldots, j_{0}\right\}=\cup_{n \in N_{0}}\left\{\tau_{1 n_{j}}\right\}=J .
$$

If $j_{0}$ is finite, then define $\tau_{1 j_{0}+1}=\infty$. It is easy to see that $S_{0}\left(\tau_{10}\right)=0$ and $\frac{d S_{0}\left(\tau_{10}\right)}{d \tau_{1}}>0$. Moreover, a Hopf bifurcation occurs when $\tau_{1}=\tau_{1 n_{j}}, j=0,1, \ldots, j_{0}$.

The function $S_{n}\left(\tau_{1}\right)$ is given by:

$$
S_{n}\left(\tau_{1}\right)=\tau_{1}-\frac{\arccos \left(-\frac{\alpha_{1}}{\beta_{1} \exp \left(-b_{3} \tau_{1}\right)}\right)+2 n \pi}{\sqrt{\beta_{1}^{2} \exp \left(-2 b_{3} \tau_{1}\right)-\alpha_{1}^{2}}}, \quad n \in N_{0} .
$$

The analysis of $S_{n}\left(\tau_{1}\right)$ can be done with the help of the graphic $\left(\tau_{1}, S_{n}\left(\tau_{1}\right)\right)$.

For the analysis of the equations (4.6), (4.7) we recall some results on the roots of the transcendental equation

$$
\lambda-a-b e^{-\lambda \tau}=0 .
$$

(see for example [7])

Theorem 9. ([9], [11]) A necessary and sufficient condition in order that all roots of Eq. (4.24) have negative real parts is that: (i) $a \tau<1$ (ii) $a \tau<-b \tau<\left(\theta^{2}+a^{2} \tau^{2}\right)^{1 / 2}$ where $\theta$ is the unique root of $\theta=\operatorname{artg}(\theta)$.

From the conditions (i) and (ii) we must have:

$$
a<\frac{1}{\tau}, a+b<0
$$

and also

$$
-b \tau<\left(\theta^{2}+a^{2} \tau^{2}\right)^{1 / 2}
$$

From (4.24), if $b>0$ and $a+b \geq 0$ or if $a \geq \frac{1}{\tau}$, there is instability. If $b<0$, the stability conditions
are:

$$
a<\frac{1}{\tau}, a<|b|<\left|a^{2}+(\theta / \tau)^{2}\right|^{1 / 2} .
$$


We analyze first the roots of equation (4.6).

For $e_{2}<e_{1}+\frac{e_{3} e_{5}}{e_{6}}$ we obtain $a_{66}+b_{663}<0$ for any $\tau_{3} \geq \frac{\ln 2}{e_{1}}$. Then (4.6) has roots with negative real part for any $\tau_{3} \geq \frac{\ln 2}{e_{1}}$.

In a similar way, for the roots of equation (4.7), we have that, for $e_{7}<1+e_{4}$, it follows that $a_{77}+b_{774}<0$ for any $\tau_{4} \geq \frac{\ln 2}{e_{4}}$. Then equation (4.7) has roots with negative real part for any $\tau_{4} \geq \frac{\ln 2}{e_{4}}$.

From the above analysis we have:

Theorem 10. If the conditions $e_{2}<e_{1}+e_{3} e_{5} / e_{6}, e_{7}<1+e_{4}$ hold true, then the stability of $E_{0}$ will depend on $\tau_{1}$. If for $\tau_{1}=0$ the root of (4.10) is negative and $\varphi_{1}(0)<0$, the real part of roots of (4.8) is negative for $\tau_{1} \in\left[0, \tau_{1}^{0}\right), \tau_{3} \geq \frac{\ln 2}{e_{1}}, \tau_{4} \geq \frac{\ln 2}{e_{4}}$ and the equilibrium $E_{0}$ of system (2.1) is locally asymptotically stable. If the root of (4.10) is positive, a change to stability can happen if the sign of $\varphi_{1}$ is negative when the real part of the roots become zero.

In order to extend the study of stability of $E_{0}$ to other situations that are not covered by Theorem 10 , Theorem 9 will be used for (4.8).

So, consider the transcendental equation

$$
\lambda+\alpha+\beta e^{-c \tau} e^{-\lambda \tau}=0
$$

thus

$$
a=-\alpha, b=-\beta e^{-c \tau}
$$

and $c>0$.

Applying Theorem 9 for (4.28) we have: Proposition 11. A necessary and sufficient condition in order that all roots of Eq. (4.28) have negative real parts is that:

$$
\begin{aligned}
& \text { (i) } \alpha>-\frac{1}{\tau} \text { and } \\
& \text { (ii) } \alpha+\beta e^{-c \tau}>0, \beta e^{-c \tau} \tau<\left(\theta^{2}+\alpha^{2} \tau^{2}\right)^{1 / 2} .
\end{aligned}
$$

From conditions (4.30), if $\beta<0$, conditions (i) and (ii) lead to:

$$
\alpha>-\frac{1}{\tau}, \alpha+\beta e^{-c \tau}>0 .
$$

Hence, if $\beta<0$ and $\alpha+\beta e^{-c \tau} \leq 0$ or $\alpha \leq-\frac{1}{\tau}$ there is instability.

If $\beta>0$, the stability conditions are:

$$
\alpha>-\frac{1}{\tau},-\alpha<\beta<\left|\alpha^{2}+\left(\theta^{2} / \tau\right)^{2}\right|^{2} .
$$

Now, we determine the stability or instability condition for $\tau$, in the conditions $\alpha>0, \beta<0$. Proposition 12. Consider $\beta<0, \alpha>0$.

(i) If $\alpha+\beta<0$, then for any $\tau>\bar{T}$, the stability conditions hold, where

$$
\bar{T}=\frac{1}{c} \ln \left(-\frac{\beta}{\alpha}\right)
$$

(ii) If $\alpha+\beta<0$, then for any $0<\tau \leq \bar{T}$, the instability condition holds.

Proof. (i) For $\beta<0, \alpha>0$, the stability condition is $\alpha+\beta e^{-c \tau}>0$. Then, $\tau>\frac{1}{c} \ln \left(-\frac{\beta}{\alpha}\right)=\bar{T}$. From the condition $\alpha+\beta<0$ we have $\bar{T}>0$. (ii) For $\beta<0, \alpha>0$, the instability condition is $\alpha+\beta e^{-c \tau} \leq 0$. Then, $0<\tau \leq \frac{1}{c} \ln \left(-\frac{\beta}{\alpha}\right)=\bar{T}$. 
Using Proposition 12, we can analyze the equation (4.8).

Consider (4.8) with $0<b_{1}<1,0<b_{2}<1,0<b_{1}+b_{2}<1$ and $\tau_{1}>\frac{1}{b_{3}} \ln 2$.

Proposition 13. If $\left(1-b_{1}-b_{2}\right) a_{3}-b_{2} a_{4}>0$ and $a_{5}<\left(1-b_{1}-b_{2}\right) a_{3}-b_{2} a_{4}$, then the solutions of (4.8) have negative real parts for any $\tau_{1}>0$.

Proof. From (4.8), we have $\beta_{1}<0, \alpha_{1}>0$ and

$$
\alpha_{1}+\beta_{1}=a_{5}+b_{2} a_{4}-\left(1-b_{1}-b_{2}\right) a_{3} .
$$

From the hypothesis, it follows that $\alpha_{1}+\beta_{1}<0$. From (4.33), we have:

$$
\bar{T}_{1}=\frac{1}{b_{3}} \ln \left(-\frac{\beta_{1}}{\alpha_{1}}\right)=\frac{1}{b_{3}} \ln \left(-\frac{2\left(b-b_{1}-b_{2}\right) a_{3}+a_{4} b_{1}}{a_{5}+\left(b_{1}+b_{2}\right) a_{4}+\left(1-b_{1}-b_{2}\right) a_{3}}\right)<\frac{1}{b_{3}} \ln 2 .
$$

Because $\tau_{1}>0$, the stability condition holds.

The reasonings above lead to

Theorem 14. Suppose that the following conditions hold true:

$$
\begin{aligned}
& \left(1-b_{1}-b_{2}\right) a_{3}-b_{2} a_{4}>0 \\
& a_{5}<\left(1-b_{1}-b_{2}\right) a_{3}-b_{2} a_{4}, \\
& e_{2}<e_{1}+e_{3} e_{5} / e_{6} \\
& e_{7}<1+e_{4}
\end{aligned}
$$

then the equilibrium point $E_{0}$ is locally asymptotically stable for all $\tau_{1}>0, \tau_{3}>\frac{\ln 2}{e_{1}}, \tau_{4}>\frac{\ln 2}{e_{4}}$.

\section{Stability analysis and existence of Hopf bifurcation for the equilibrium points $E_{1}$ and $E_{2}$}

Let $E_{1}$ be the equilibrium point with the coordinates given by (3.4) in the conditions of Proposition 2 .

Consider the transformation $u_{1}(t)=x_{1}(t), u_{2}(t)=x_{2}(t), u_{3}(t)=x_{3}(t)-\frac{c_{1}}{c_{2}}, u_{4}(t)=x_{4}(t), u_{5}(t)=$ $x_{5}(t)-\frac{d_{3}}{d_{4}}, u_{6}(t)=x_{6}(t)-x_{6}^{*}, u_{7}(t)=x_{7}(t)$.

The linearized system, obtained from (2.1) with the above transformation, is given by:

$$
\begin{aligned}
& \dot{u}_{1}(t)=a_{11} u_{1}(t)+b_{111} u_{1}\left(t-\tau_{1}\right), \\
& \dot{u}_{2}(t)=a_{22} u_{2}(t)+b_{212} u_{1}\left(t-\tau_{2}\right), \\
& \dot{u}_{3}(t)=a_{31} u_{1}(t)+a_{32} u_{2}(t)+a_{33} u_{3}(t), \\
& \dot{u}_{4}(t)=a_{41} u_{1}(t)+a_{42} u_{2}(t)+a_{44} u_{4}(t), \\
& \dot{u}_{5}(t)=a_{54} u_{4}(t)+a_{55} u_{5}(t), \\
& \dot{u}_{6}(t)=c_{66} u_{6}(t)+d_{663} u_{6}\left(t-\tau_{3}\right)+d_{645} u_{4}\left(t-\tau_{5}\right) \\
& \dot{u}_{7}(t)=c_{77} u_{7}(t)+d_{746} u_{4}\left(t-\tau_{6}\right)+d_{774} u_{7}\left(t-\tau_{4}\right)
\end{aligned}
$$

where $a_{11}, a_{22}, a_{31}, a_{41}, a_{54}, b_{111}, b_{212}, a_{32}, a_{33}, a_{42}, a_{44}, a_{55}, b_{746}$ are given by $(4.2)$ and

$$
\begin{aligned}
& c_{66}=-e_{1}-e_{2}\left(\zeta_{1}^{\prime}\left(x_{6}^{*}\right) x_{6}^{*}+\zeta_{1}\left(x_{6}^{*}\right)\right)-e_{3}\left(\zeta_{2}^{\prime}\left(x_{6}^{*}\right) x_{6}^{*}+\zeta_{2}\left(x_{6}^{*}\right)\right) \\
& d_{663}=2 e^{-e_{1} \tau_{3}} e_{2}\left(\zeta_{1}^{\prime}\left(x_{6}^{*}\right) x_{6}^{*}+\zeta_{1}\left(x_{6}^{*}\right)\right), d_{645}=2^{m_{1}} \frac{d_{61} d_{3}}{d_{4}} \\
& c_{77}=-1-e_{4}-e_{7} \zeta_{3}\left(x_{6}^{*}\right), d_{774}=2 e_{7} e^{-e_{4} \tau_{4}} \zeta_{3}\left(x_{6}^{*}\right)
\end{aligned}
$$


The characteristic equation of system (5.1) is:

$$
\begin{aligned}
& \left(\lambda-a_{11}-b_{111} e^{-\lambda \tau_{1}}\right)\left(\lambda-a_{22}\right)\left(\lambda-a_{33}\right)\left(\lambda-a_{44}\right)\left(\lambda-a_{55}\right) . \\
& \cdot\left(\lambda-c_{66}-d_{663} e^{-\lambda \tau_{3}}\right)\left(\lambda-c_{77}-d_{774} e^{-\lambda \tau_{4}}\right)=0 .
\end{aligned}
$$

The roots of (5.3) are given by:

$$
\lambda=a_{22}=-b_{6}, \lambda_{3}=a_{33}=-c_{3}, \lambda_{4}=a_{44}=-d_{2}, \lambda_{5}=a_{55}=-d_{4},
$$

and by the solutions of the equations:

$$
\begin{aligned}
& \lambda-a_{11}-b_{111} e^{-\lambda \tau_{1}}=0 \\
& \lambda-c_{66}-d_{663} e^{-\lambda \tau_{3}}=0 \\
& \lambda-c_{77}-d_{774} e^{-\lambda \tau_{4}}=0
\end{aligned}
$$

The analysis of the roots for equation (5.4) has been done in the previous section, with the conditions $0 \leq \tau_{1}<\tau_{11}$. In what follows, we analyze the roots of Eq. (5.5) using the method from the previous section.

Consider

$$
\begin{aligned}
& \gamma_{60}=-c_{66}=e_{1}+e_{2}\left(\zeta_{1}^{\prime}\left(x_{6}^{*}\right) x_{6}^{*}+\zeta_{1}\left(x_{6}^{*}\right)\right)+e_{3}\left(\zeta_{2}^{\prime}\left(x_{6}^{*}\right) x_{6}^{*}+\zeta_{2}\left(x_{6}^{*}\right)\right) \\
& \delta_{60}=-2 e_{2}\left(\zeta_{1}^{\prime}\left(x_{6}^{*}\right) x_{6}^{*}+\zeta_{1}\left(x_{6}^{*}\right)\right) .
\end{aligned}
$$

Then, equation (5.5) becomes:

$$
\lambda+\gamma_{60}+\delta_{60} e^{-e_{1} \tau_{3}} e^{-\lambda \tau_{3}}=0
$$

Let $p_{2}=2$.

Eq. (3.5) can be written as $f\left(x_{6}\right)=0$, where

$$
f\left(x_{6}\right)=m_{2} x_{6}^{4}+m_{1} x_{6}^{2}+m_{0}
$$

where

$$
\begin{aligned}
& m_{2}=e_{1}+e_{3}, m_{1}=e_{1}+e_{3}+e_{1} e_{6}+e_{3} e_{5}+\left(1-2 e^{-e_{1} \tau_{3}}\right) e_{2}, \\
& m_{0}=e_{1} e_{6}+e_{3} e_{5}+e_{2} e_{6}\left(1-2 e^{-e_{1} \tau_{3}}\right),
\end{aligned}
$$

$T_{30} \leq \tau_{3}<\ln 2 / e_{1}, T_{30}, T_{31}$ are given by (3.3). Deriving $f\left(x_{6}\right)$ with respect to $x_{6}$ we have:

$$
f^{\prime}\left(x_{6}\right)=4 m_{2} x_{6}^{3}+2 m_{1} x_{6} .
$$

Because $m_{2}>0, m_{1}>0$ then $f^{\prime}\left(x_{6}\right) \geq 0$ for any $x_{6} \geq 0$. Then, $f\left(x_{6}\right)$ is an increasing function for $x_{6} \geq 0$. From $f(0)=m_{0}<0$ and $m_{2}>0$, we obtain that the equation $f\left(x_{6}\right)=0$ has a unique positive root. Let $x_{6}^{*}=x_{6}^{*}\left(\tau_{3}\right)$ be the positive root.

From (5.7), with $\zeta_{1}$ given by (2.2) we have:

$$
\begin{aligned}
\gamma_{60} & =e_{1}-\frac{e_{2}\left(x_{6}^{* 2}-1\right)}{\left(x_{6}^{* 2}+1\right)^{2}}+\frac{e_{6}\left(x_{6}^{* 4}+\left(3 e_{6}-e_{5}\right) x_{6}^{* 2}+e_{5} e_{6}\right)}{\left(x_{6}^{* 2}+e_{6}\right)^{2}} \\
\delta_{60} & =2 \frac{e_{2}\left(x_{6}^{* 2}-1\right)}{\left(x_{6}^{* 2}+1\right)^{2}}
\end{aligned}
$$

From (5.11), we have:

Proposition 15. The following statements hold true: 
1. $x_{6}^{*}\left(\tau_{3}\right)>1$ for $\tau_{3} \in\left[T_{30}, T_{32}\right)$, where

$$
T_{32}=\frac{1}{e_{1}} \ln \frac{2 e_{2}\left(1+e_{6}\right)}{2\left(e_{1}+e_{3}+e_{1} e_{6}+e_{3} e_{5}\right)+e_{2}\left(1+e_{6}\right)}
$$

and $T_{32} \leq T_{31}$.

2. If $e_{6}>e_{5}, e_{1}>\frac{3 e_{2}}{2}$ and $\tau_{3} \in\left[T_{30}, T_{32}\right)$ then,

$$
\begin{aligned}
\gamma_{60}+\delta_{60} & >0 \\
\gamma_{60}-\delta_{60} & >0,
\end{aligned}
$$

where $T_{30}$ is given by (3.3) and $T_{32}$ is given by (5.12).

Proof. From (5.9) and (5.10) we have:

$$
f(1)=2\left(e_{1}+e_{3}+e_{1} e_{6}+e_{3} e_{5}\right)+e_{2}\left(1+e_{6}\right)-2 e_{2}\left(1+e_{6}\right) e^{-e_{1} \tau_{3}} .
$$

For $\tau_{3} \in\left[T_{30}, T_{32}\right)$, with $T_{32}$ given by (5.12), we obtain that $f(1)<0$. Thus, $x_{6}^{*}\left(\tau_{3}\right)>1$.

From (5.11) we obtain (5.13). From (5.11), it follows that:

$$
\gamma_{60}-\delta_{60}=\frac{e_{1}\left(x_{6}^{*}\right)^{4}+\left(2 e_{1}-3 e_{2}\right)\left(x_{6}^{*}\right)^{2}+e_{1}+3 e_{2}}{\left(\left(x_{6}^{*}\right)^{2}+1\right)^{2}}+\frac{e_{6}\left(\left(x_{6}^{*}\right)^{4}+\left(3 e_{6}-e_{5}\right)\left(x_{6}^{*}\right)^{2}+e_{5} e_{6}\right)}{\left(\left(x_{6}^{*}\right)^{2}+e_{6}\right)^{2}} .
$$

Because $2 e_{1}>3 e_{2}$ and $e_{6}>e_{5}$, the relation (5.14) holds true.

From Proposition 15, Eq. (5.8) has roots with negative real part for any $\tau_{3} \in\left[T_{30}, T_{32}\right.$ ). For Eq. (5.8) there is no Hopf bifurcation with respect to $\tau_{3}$.

Using Proposition 12 we analyze (5.6). We use:

$$
\gamma_{70}=1+e_{4}+e_{7} \zeta_{3}\left(x_{6}^{*}\right), \delta_{70}=-2 e_{7} \zeta_{3}\left(x_{6}^{*}\right)
$$

Proposition 16. If $e_{7}>\left(1+e_{4}\right) / \zeta_{3}\left(x_{6}^{*}\right)$ the stability condition holds for any $\tau_{4}>\frac{1}{e_{4}} \ln 2$.

Proof. From (5.15), we have $\delta_{70}<0, \gamma_{70}>0$.

From the hypothesis it follows that $\gamma_{70}+\delta_{70}<0$ and from (5.6) we obtain:

$$
\bar{T}=\frac{1}{e_{4}} \ln \left(\frac{2 e_{7} \zeta_{3}\left(x_{6}^{*}\right)}{1+e_{4}+e_{7} \zeta_{3}\left(x_{6}^{*}\right)}\right)<\frac{1}{e_{4}} \ln 2 .
$$

Because $\bar{T}<\frac{1}{e_{4}} \ln 2$, the stability condition holds for any $\tau_{4}>\frac{1}{e_{4}} \ln 2$.

In this case there does not exist a Hopf bifurcation with respect to $\tau_{4}$.

The following theorem has been proved:

Theorem 17. Suppose that the delays $\tau_{1}, \tau_{3}, \tau_{4}$ satisfy the conditions: $E_{1}$.

$\tau_{1}>0, T_{30} \leq \tau_{3}<T_{32}, \tau_{4}>\frac{\ln 2}{e_{4}}$, where $T_{32}$ is given by (5.12). Then, there exists the equilibrium point

If the following conditions hold:

$$
\begin{aligned}
& \left(1-b_{1}-b_{2}\right) a_{3}-b_{2} a_{4}>0, \\
& a_{5}<\left(1-b_{1}-b_{2}\right) a_{3}-b_{2} a_{4}, \\
& e_{2}>e_{1}, \\
& e_{6}>\max \left(e_{5} / 3, e_{3} e_{5} /\left(e_{2}-e_{1}\right)\right), \\
& e_{7}>\left(1+e_{4}\right)\left(1+\left(x_{6}^{*}\right)^{p_{3}}\right)
\end{aligned}
$$


then the equilibrium point $E_{1}$ is locally asymptotically stable.

For the analysis of the equilibrium point $E_{2}$, we consider the transformation $u_{1}(x)=x_{1}(t), u_{2}(t)=$ $x_{2}(t), u_{3}(t)=x_{3}(t)-c_{1} / c_{2}, u_{4}(t)=x_{4}(t), u_{5}(t)=x_{5}(t)-d_{3} / d_{4}, u_{6}(t)=x_{6}(t)-x_{61}, u_{7}(t)=x_{7}(t)-c$, where $x_{61}=x_{6}^{*}\left(\tau_{3}\right)$ is a positive root of equation (3.5) and $c>0$.

The linearized system, obtained by this translation, is given by (5.1), with $\tau_{4}\left(\tau_{3}\right)=T_{40}$ given by (3.8). The analysis of equations $(5.4),(5.5)$ is the same as the case of the equilibrium point $E_{1}$. We analyze eq. (5.6) with $\zeta_{3}\left(x_{6}^{*}\right)=\frac{1}{1+\left(x_{6}^{*}\right)^{2}}$ for $\tau_{4}\left(\tau_{3}\right)$ and $\tau_{3} \in\left[T_{30}, T_{31}\right)$.

Consider the function $\tau_{4}\left(\tau_{3}\right)$ given by:

$$
\tau_{4}\left(\tau_{3}\right)=\frac{1}{e_{4}} \ln \frac{2 e_{7}}{\left(1+e_{4}\right)\left(1+x_{6}^{*}\left(\tau_{3}\right)^{2}\right)+e_{7}} .
$$

Proposition 18. For $e_{7}>1+e_{4}$, define $x_{0}=\frac{e_{7}-1-e_{4}}{1+e_{4}}$,

$$
A=\frac{2 e_{2}\left(x_{0}+e_{6}\right)}{\left(e_{1}+e_{3}\right) x_{0}^{2}+\left(e_{1}+e_{2}+e_{3}+e_{1} e_{6}+e_{3} e_{5}\right) x_{0}+e_{1} e_{6}+e_{3} e_{5}+e_{2} e_{6}}
$$

and also

$$
T_{33}=\frac{1}{e_{1}} \ln A .
$$

Then the following statements hold true:

$$
\tau_{4}\left(T_{33}\right)=0 \quad \text { and } \quad T_{33}<T_{31} .
$$

Proof. From (5.16), if $\tau_{4}\left(\tau_{3}\right)=0$ then $x_{6}^{*}\left(\tau_{3}\right)=x_{0}$. Because $x_{6}^{*}\left(\tau_{3}\right)$ is the solution of the equation (5.9) then $f\left(x_{0}\right)=0$. Using (5.9) and (5.10) we obtain:

$$
2 e_{2}\left(x_{0}+e_{6}\right) e^{-e_{1} \tau_{3}}=\left(e_{1}+e_{3}\right) x_{0}^{2}+\left(e_{1}+e_{2}+e_{3}+e_{1} e_{6}+e_{3} e_{5}\right) x_{0}+e_{1} e_{6}+e_{3} e_{5}+e_{2} e_{6} .
$$

Therefore, $\tau_{3}=T_{33}$ where $T_{33}$ is given by (5.18). Thus, $\tau_{4}\left(T_{33}\right)=0$.

Eq. (5.6) can be written as:

$$
\begin{gathered}
\lambda+\gamma_{70}+\delta_{70} e^{-e_{4} \tau_{4}\left(\tau_{3}\right)} e^{-\lambda \tau_{4}\left(\tau_{3}\right)}=0 \\
\gamma_{70}=1+e_{4}+\frac{e_{7}}{1+\left(x_{6}^{*}\left(\tau_{3}\right)\right)^{2}}, \delta_{70}=-\frac{2 e_{7}}{1+\left(x_{6}^{*}\left(\tau_{3}\right)\right)^{2}} .
\end{gathered}
$$

For $\tau_{3}=T_{33}$, because $\tau_{4}\left(T_{33}\right)=0$, from (5.19), we have:

$$
\lambda+\gamma_{70}+\delta_{70}=0
$$

Because

$$
\gamma_{70}+\delta_{70}=1+e_{4}-\frac{e_{7}}{1+x_{0}^{2}}<0
$$

equation (5.19) does not have roots with negative real part.

In this case does not exist a Hopf bifurcation and the equilibrium point $E_{2}$ is unstable.

From the analysis above the following theorem is proved

Theorem 19. The equilibrium point $E_{2}$ of system (2.1) is unstable. 


\section{Numerical simulations}

The numerical simulations are done using first the following values for the parameters, most of them being extracted from existing literature: for the parameters related to leukopoiesis (the first two equations) we rely on [5], [10], while those of the immune system were taken, when possible, from [17], [18]. The parameters, used in the feedback functions, were estimated on the basis of existing reports on the number of specific cells of the immune system. The rates of asymmetric and symmetric division were chosen according to the observation in [28] that in leukemia symmetric division is dominant.

\begin{tabular}{|c|c|c|}
\hline Parameter for the $\beta$ function & $a_{1}$ & 0.5 \\
\hline Parameter for the function $k$ & $a_{2}$ & 36 \\
\hline The maximum value of the $\beta$ function & $a_{3}$ & 1.77 \\
\hline The maximum value of the function $k$ & $a_{4}$ & 0.8 \\
\hline $\begin{array}{l}\text { Loss of stem cells due to mortality and differentiation in the eritrocyte } \\
\text { and platelets lines }\end{array}$ & $a_{5}$ & 0.12 \\
\hline Rate of asymmetric division & $b_{1}$ & 0.1 \\
\hline Rate of symmetric division & $b_{2}$ & 0.4 \\
\hline Loss of stem cells during cell cycle & $b_{3}$ & 0.02 \\
\hline Loss of stem cells due to cytotoxic T cells & $b_{4}$ & 0.03 \\
\hline Depends on individual case & $b_{5}$ & 6 \\
\hline Instant mortality of mature leukocytes & $b_{6}$ & 2.4 \\
\hline Amplification factor for leukocytes & $b_{7}$ & 1440 \\
\hline Loss of mature leukocytes due to cytotoxic T cells & $b_{8}$ & 0.6 \\
\hline Coefficient expressing both apopthosis rate and regulatory mechanism & $b_{9}$ & 0.7 \\
\hline Supply rate of immature APCs, $c_{2} x_{3}(0)$ & $c_{1}$ & $0.3 /$ day \\
\hline Death/turnover rate of immature APCs & $c_{2}$ & $0.03 /$ day \\
\hline Coefficient of the feedback function & $c_{3}$ & 2 \\
\hline Death/turnover rate of mature APCs & $d_{2}$ & $0.8 /$ day \\
\hline Supply rate of naive T cells( of both CD $4+$ and CD $8+$ phenotype) & $d_{3}$ & 2 \\
\hline Death/turnover rate of naive CD4+ and CD8+ T cells & $d_{4}$ & $0.03 /$ day \\
\hline Kinetic coefficient & $d_{5}$ & 0.2 \\
\hline Kinetic coefficients & $d_{61}, d_{62}$ & $0.12,0.08$ \\
\hline Death/turnover rate of effector CD4+ T helper cells & $e_{1}$ & $0.23 /$ day \\
\hline Coefficient of the "autocrine loop" function, $\zeta_{1}\left(x_{6}(t)\right)$ & $e_{2}$ & $\begin{array}{l}0.2\left(\text { for } E_{0}\right) \\
50\left(\text { for } E_{1}\right)\end{array}$ \\
\hline Coefficient of the "regulatory process" function, $\zeta_{2}(x 6(t))$ & $e_{3}$ & 1 \\
\hline Death/turnover rate of effector CD8+ T cytotoxic cells & $e_{4}$ & $0.5 /$ day \\
\hline Coefficient of the "regulatory process" function, $\zeta_{2}(x 6(t))$ & $e_{5}$ & 2600 \\
\hline Coefficient of the "regulatory process" function, $\zeta_{2}(x 6(t))$ & $e_{6}$ & 260000 \\
\hline Coefficient of the "positive growth signal" function, $\zeta_{3}(x 6(t))$ & $e_{7}$ & $\begin{array}{l}1.2\left(\text { for } E_{0}\right) \\
50\left(\text { for } E_{1}\right)\end{array}$ \\
\hline Coefficient of the suppressive action of the leukemia cells & $e_{8}$ & 5 \\
\hline Coefficient of the level of down-regulation due to leukemic cells & $e_{9}$ & 0.4 \\
\hline The number of antigen depending divisions & $n_{1}$ & 2 \\
\hline Number of divisions in minimal CD4+ developmental program & $m_{1}$ & 2 \\
\hline Number of divisions in minimal CD8+ developmental program & $m_{2}$ & 7 \\
\hline Coefficient of the feedback function & $\mathrm{p}$ & 2 \\
\hline Coefficient of the "autocrine loop" function, $\zeta_{1}(x 6(t))$ & $p_{2}$ & 2 \\
\hline Coefficient of the "positive growth signal" function, $\zeta_{3}(x 6(t))$ & $p_{3}$ & 2 \\
\hline Bifurcation parameter & $\tau_{1}$ & 5.5 \\
\hline Duration of leukocyte cycle & $\tau_{2}$ & 5.4 \\
\hline Duration of one CD4+ T cell division (bifurcation parameter) & $\tau_{3}$ & $\begin{array}{l}3.6 \text { days (for } E_{0} \text { ), } \\
2.4 \text { days (for } E_{1} \text { ) }\end{array}$ \\
\hline Duration of one CD8+ T cell division & $\tau_{4}$ & 1.4 days \\
\hline Duration of minimal developmental program, $1+\left(m_{1}-1\right) \tau_{3}$ & $\tau_{5}$ & 4.6 \\
\hline Duration of minimal developmental program, $1+\left(m_{2}-1\right) \tau_{4}$ & $\tau_{6}$ & 8.4 \\
\hline Initial concentration of immature APCs & $x_{3}(0)$ & $c_{1} / c_{2}+0.00001$ \\
\hline $\begin{array}{l}\text { Initial naive } \mathrm{T} \text { cells (of both CD4+ and } \\
\text { CD8+ phenotype) concentration }\end{array}$ & $x_{5}(0)$ & $d_{3} / d_{4}+0.00001 /$ day \\
\hline
\end{tabular}

FIgURE 1. The concentrations are given in units of $k / \mu L$, and the time is measured in days. 


\subsection{A numerical simulation in the neighborhood of $E_{0}$}

From the above table we consider the following parameters: $a_{3}=1.77, a_{4}=0.8, a_{5}=0.12, b_{1}=0.1$, $b_{2}=0.4, b_{3}=0.2$. We obtain:

$$
\begin{aligned}
& \left(1-b_{1}-b_{2}\right) a_{3}-b_{2} a_{4}=0.565>0, \\
& a_{5}-\left(1-b_{1}-b_{2}\right) a_{3}-b_{2} a_{4}=-1.085<0,
\end{aligned}
$$

With $e_{1}=0.23, e_{2}=0.2, e_{3}=1, e_{5}=2600, e_{6}=260000$ we have: $e_{2}-e_{1}-e_{3} e_{5} / e_{6}=-0.04<0$, $\frac{\ln 2}{e_{1}}=3.013$.

With $e_{4}=0.5, e_{7}=1.2$ we have: $e_{7}-1-e_{4}=-0.3<0, T_{4}=\frac{\ln 2}{e_{4}}=1.3862$.

Thus, the conditions from Theorem 14 are satisfied.

The coordinates of the equilibrium point $E_{0}$ are: $x_{10}=0, x_{20}=0, x_{30}=10, x_{40}=0, x_{50}=66.6$, $x_{60}=0, x_{70}=0$.

Consequently, the equilibrium point $E_{0}$ is locally asymptotically stable.

With Matlab, we can visualize the orbits $\left(t, x_{i}(t)\right), i=1, . ., 7$ using the initial conditions:

$$
x_{1}(0)=\varepsilon_{1}, x_{2}(0)=\varepsilon, x_{3}(0)=x_{30}+\varepsilon, x_{4}(0)=\varepsilon, x_{5}(0)=x_{50}+\varepsilon, x_{6}(0)=\varepsilon_{1}, x_{7}(0)=\varepsilon
$$

where $\varepsilon=0.1$ and $\varepsilon_{1}=0.5$.

The values of the delays are: $\tau_{1}=5.5, \tau_{2}=5.4, \tau_{3}=3.6, \tau_{4}=1.4, \tau_{5}=4.6, \tau_{6}=8.4, \tau_{7}=2 \tau_{4}$. The interval of integration is $[0,200]$.

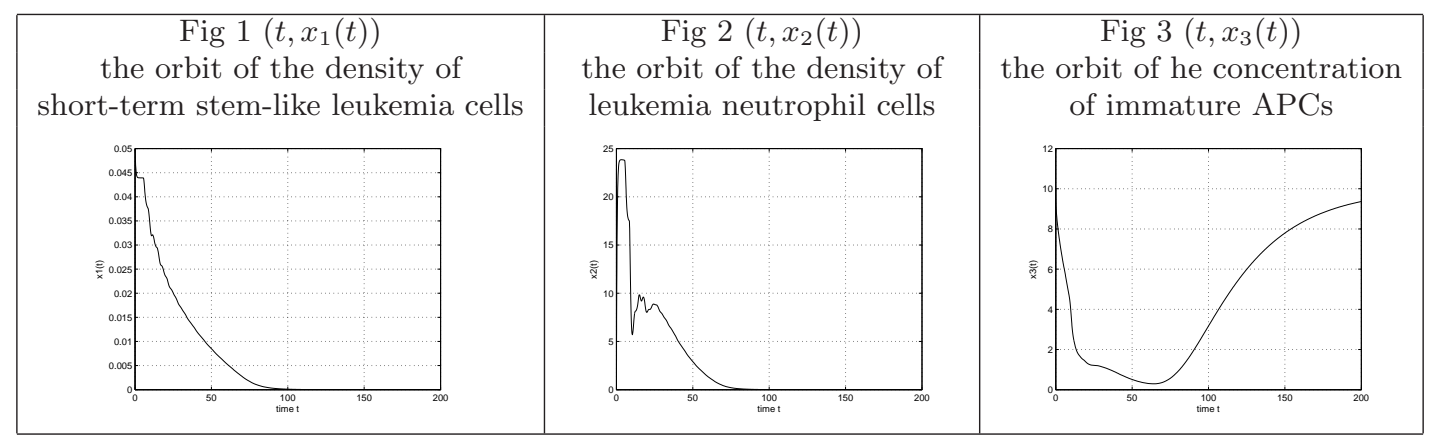

\begin{tabular}{|c|c|c|}
\hline $\begin{array}{c}\text { Fig } 4\left(t, x_{4}(t)\right) \\
\text { the orbit of the concentration } \\
\text { of mature APCs }\end{array}$ & $\begin{array}{c}\text { Fig } 5\left(t, x_{5}(t)\right) \\
\text { the orbit of the concentration } \\
\text { of passive T cells of both CD4+ } \\
\text { and CD8+ phenotype }\end{array}$ & $\begin{array}{c}\text { Fig } 6\left(t, x_{6}(t)\right) \\
\text { the orbit of the concentration } \\
\text { of active CD4+ T-helper cells }\end{array}$ \\
\hline
\end{tabular}




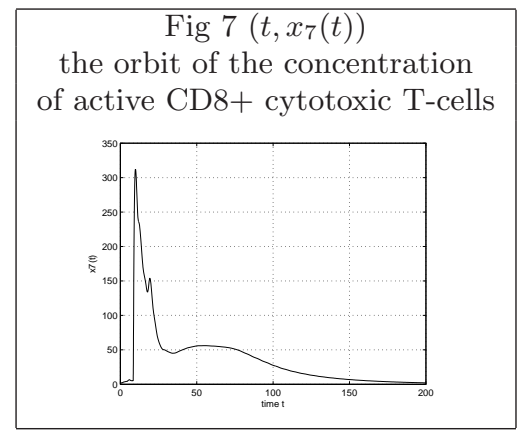

\subsection{A numerical simulation in the neighborhood of $E_{1}$}

For the values $b_{1}=0.1, b_{2}=0.4, a_{3}=1.77, a_{4}=0.8, a_{5}=0.12, b_{3}=0.02, e_{4}=0.5, e_{3}=1, e_{5}=2600$, $e_{2}=50, e_{1}=0.23, e_{6}=260000$, we have: $T_{31}=2.992863756$. Then, we choose $\tau_{3}=2.4$. The equation

$$
\left(e_{1}+e_{3}\right) x^{4}+\left(e_{1}+e_{3}+e_{1} e_{6}+e_{3} e_{5}+\left(1-2 e^{-e_{1} \tau_{3}}\right) e_{2}\right) x^{2}+e_{1} e_{2}+e_{3} e_{5}+e_{2} e_{6}\left(1-2 e^{-e_{1} \tau_{3}}\right)=0
$$

has the positive root $x_{6}^{*}=5.106259210$. The coordinates of the equilibrium point $E_{1}$ are:

$$
x_{10}=0, x_{20}=0, x_{30}=10, x_{40}=0, x_{50}=6.66, x_{60}=5.106259210, x_{70}=0 .
$$

The conditions from Theorem 17 are satisfied.

With Matlab, we can visualize the orbits $\left(t, x_{i}(t)\right), i=1 . .7$ using the initial conditions

$$
x_{1}(0)=\varepsilon_{1}, x_{2}(0)=\varepsilon, x_{3}(0)=x_{30}+\varepsilon, x_{4}(0)=\varepsilon, x_{5}(0)=x_{50}+\varepsilon, x_{6}(0)=x_{60}+\varepsilon_{1}, x_{7}(0)=\varepsilon,
$$

where $\varepsilon=0.1$ and $\varepsilon_{1}=0.5$.

The values of the delays are:

$$
\tau_{1}=5.5, \tau_{2}=5.4, \tau_{3}=2.4, \tau_{4}=1.4, \tau_{5}=4.6, \tau_{6}=8.4, \tau_{7}=2 \tau_{4} .
$$

The integration interval is $[0,200]$.

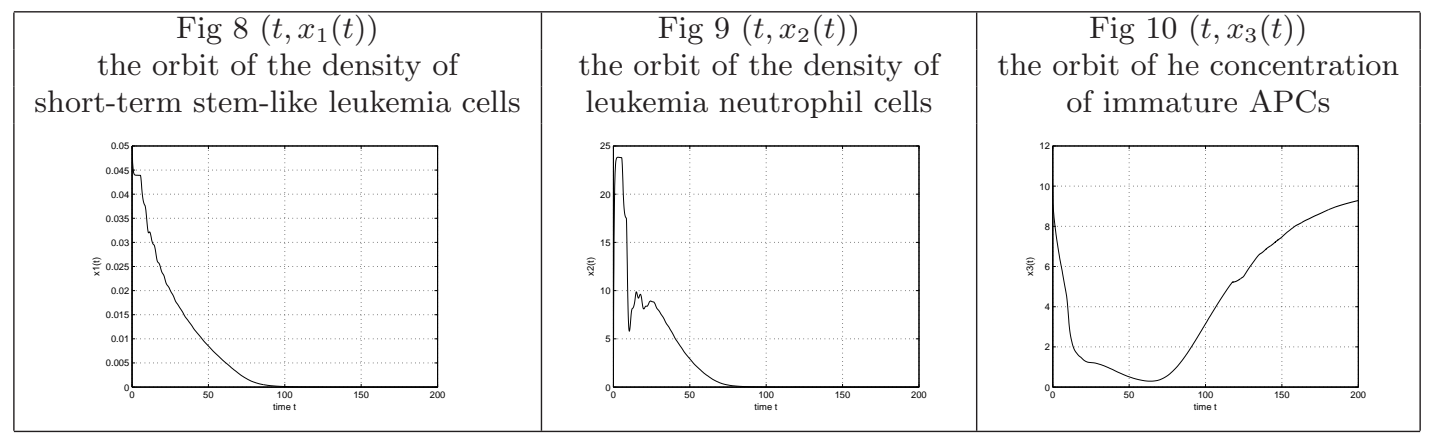




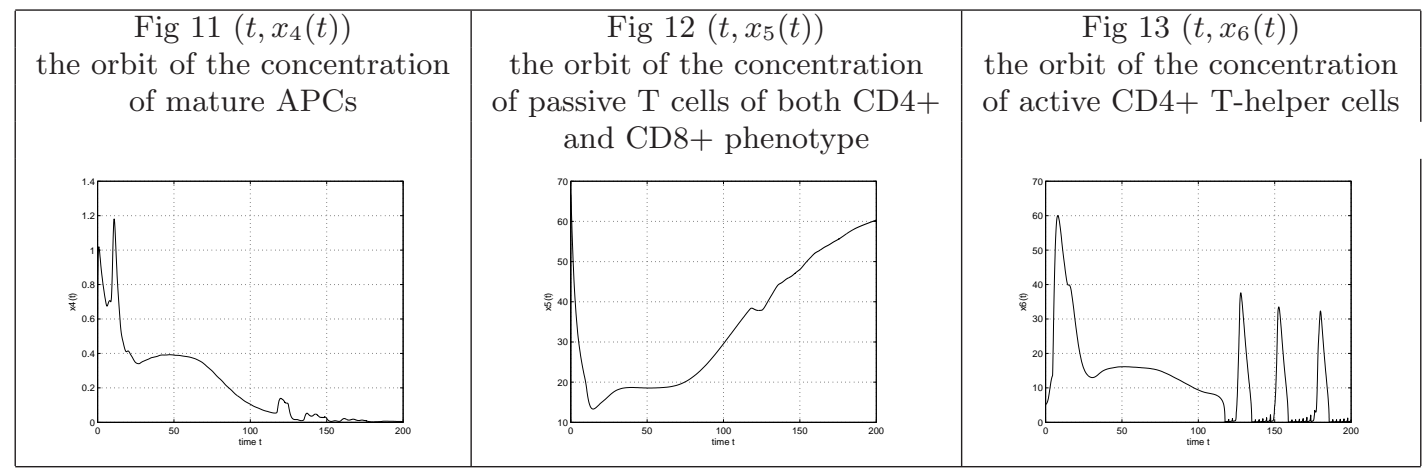

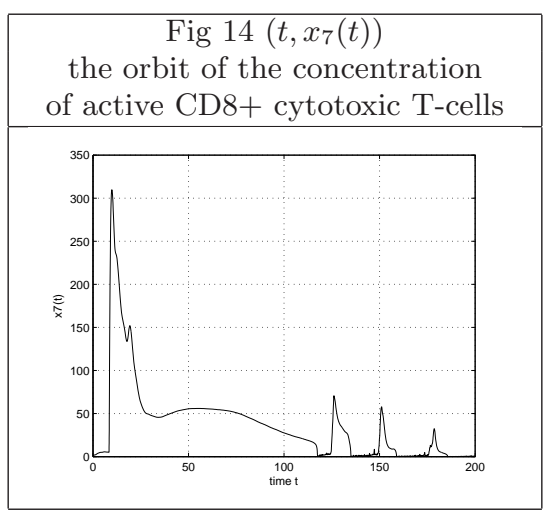

\subsection{A numerical simulation in the neighborhood of $E_{0}:$ the case of a Hopf bifurcation}

Now, we change the values of some parameters and we consider $a_{3}=0.9, a_{4}=0.9, a_{5}=0.05, b_{1}=0.1$, $b_{2}=0.4$. Then, $\alpha_{1}=0.95, \beta_{1}=-0.99$.

The graphic of the function $S_{n}\left(\tau_{1}\right)$ given by $(4.23)$ is:

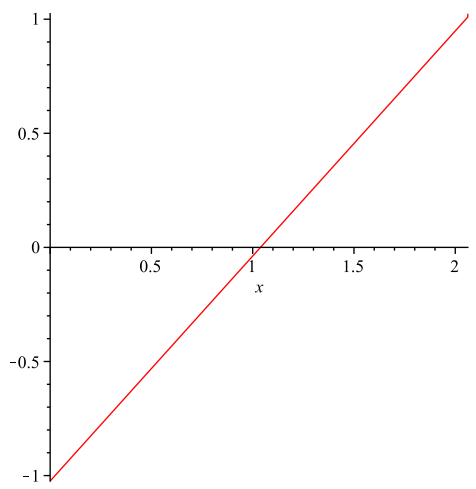

It follows that $\tau_{1}^{*}=1.02$ and $\varphi_{1}\left(\tau_{1}^{*}\right)>0$. From Theorem 10 we infer that $\tau_{1}^{*}$ is a Hopf bifurcation point. 
For $\tau_{1}=1.02, \tau_{2}=4.5, \tau_{3}=3.6, \tau_{4}=3.4, \tau_{5}=4.6, \tau_{6}=8.4, \tau_{7}=2 \tau_{4}$ and the initial conditions from the section 6.1 on the interval $[0,1050]$, we obtain the orbits $\left(t, x_{i}(t)\right), i=1 . .7$ displayed in Fig 15 -Fig 21.

The figures Fig 22-Fig 27 represent the effect between $x_{1}(t)$ and $x_{i}(t), i=2 . .7$.

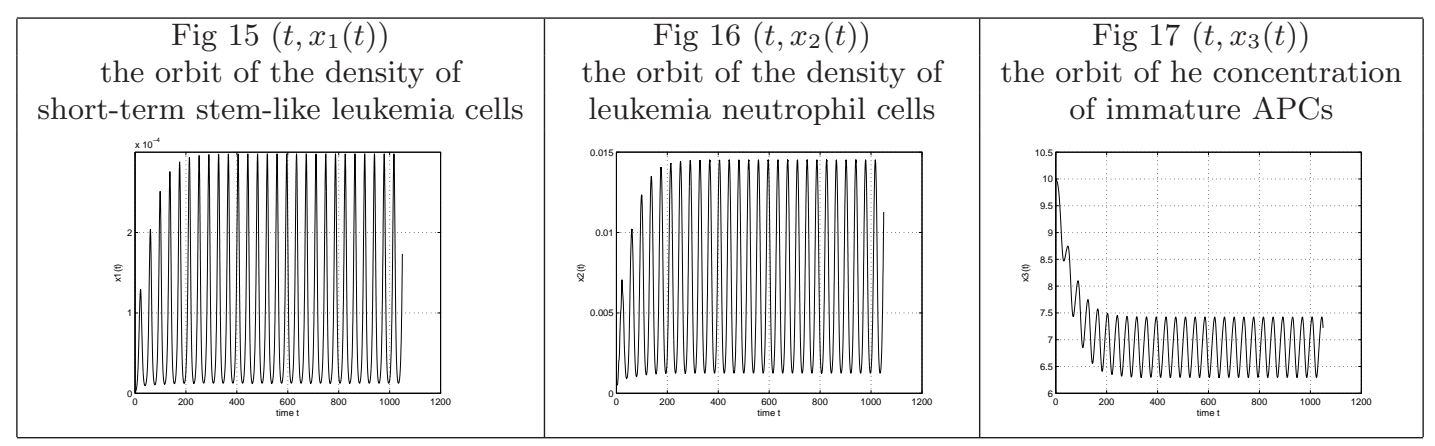

\begin{tabular}{|c|c|c|c|}
\hline $\begin{array}{c}\text { Fig 18 }\left(t, x_{4}(t)\right) \\
\text { the orbit of the concentration } \\
\text { of mature APCs }\end{array}$ & $\begin{array}{c}\text { Fig 19 }\left(t, x_{5}(t)\right) \\
\text { the orbit of the concentration } \\
\text { of passive T cells of both CD4+ } \\
\text { and CD8+ phenotype }\end{array}$ & $\begin{array}{c}\text { Fig } 20\left(t, x_{6}(t)\right) \\
\text { the orbit of the concentration } \\
\text { of active CD4+ T-helper cells }\end{array}$ \\
\hline
\end{tabular}



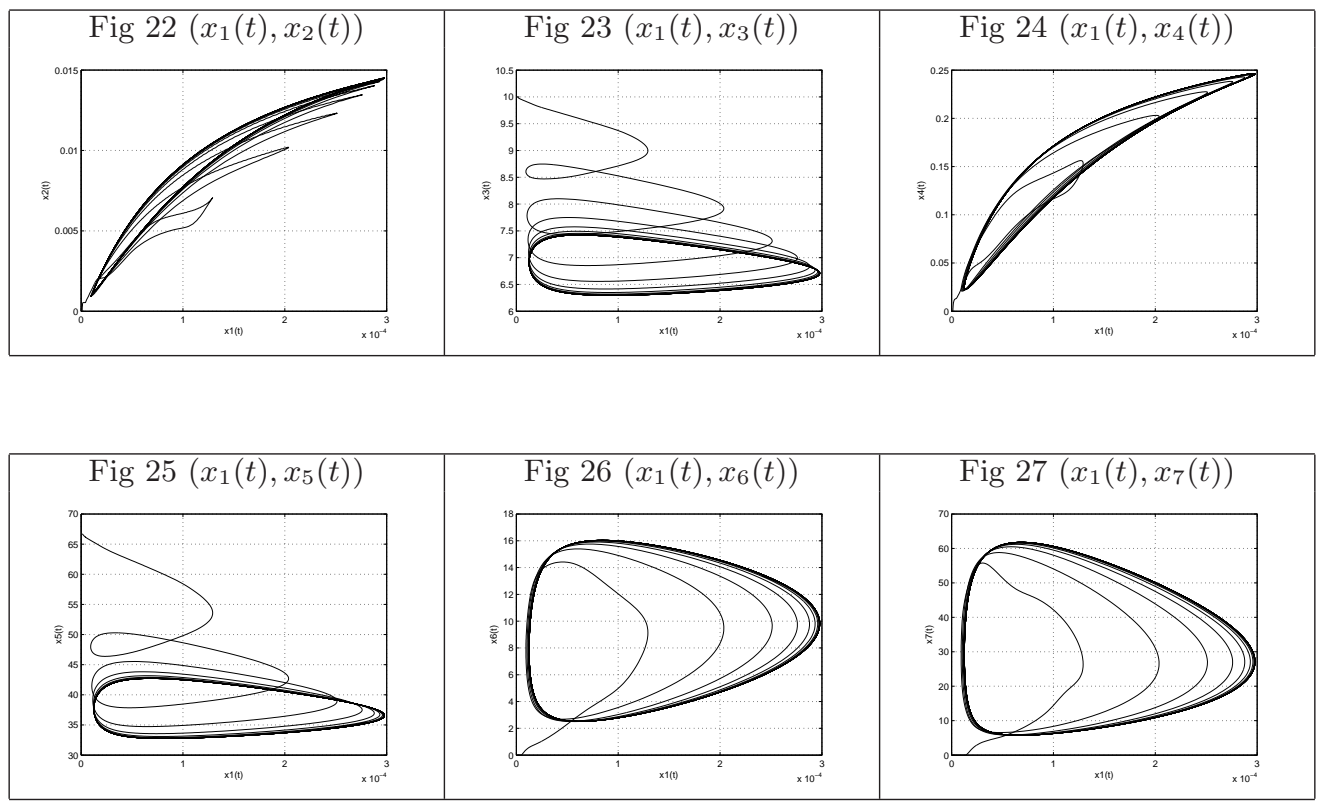

The above figures confirm the existence of the limit cycle.

\section{Conclusions}

The immune system has a crucial role in modulating tumor progression and response to therapy. In most cases the immune response is the result of multiple interactions amongst different cell types. Although the immune system has proven to provide possible strategies for the treatment of blood cancers, the full potential of immune-based therapies for these types of cancers is still unknown.

Chronic myeloid leukemia (CML) is recognized as a disease model for oncogene addiction, targeted therapy and cancer stem cells. Both innate and adaptive immunity are implicated in the defense mechanisms against tumoral antigens. The two can be thought as being equally important facets of the immune system.

The innate immune system consisting of several classes of preformed soluble molecules and cells detects and kills the antigens within minutes to hours but does not rely on clonal expansion of lymphocytes.

The adaptive immunity is somehow slower but demonstrates exquisite specificity for distinct molecules and an ability to remember and respond more vigorously to repeated exposures to the same antigen. The adaptive immune system can recognize and react to many substances. The main components of adaptive immunity are lymphocytes (B and T cells) and the antibodies. An important subset of $\mathrm{T}$ cells generated in the thymus is the regulatory $\mathrm{T}$ cells (Tregs). T helper and Tregs cells have a central role in the antitumor immune response especially because the targeted antigens are self-antigens. Recent immunotherapeutic protocols are exploring depletion or the modulation of $\mathrm{T}$ cell function in order to enhance adaptive immune responses. Also it has been hypothesized that imatinib may create a window in which the immune response is partially restored while apoptotic leukemic cells are present, thus rendering leukemic cells immunogenic as patients enter remission.

Our mathematical model covers only a small part of the various nuances of the immune response possible in a disease such as leukemia. Understanding how $\mathrm{T}$ helper cells behave during various antigen - challenges is currently of major interest in immunology. Such knowledge could be applied in the field of research for the evaluation of a drug/vaccine efficacy during preclinical phases of studies.

The model studies the natural dynamics, uninfluenced by treatment, of leukemic cells such as shortterm stem-like cells and mature leukocyte, paralleled by the evaluation of the adaptive immune response. 
The physiological processes were illustrated using a system of seven delay differential equations with seven delays leading to a highly complex mathematical model with many parameters.

The model was analyzed, steady states were found and their stability has been studied using the characteristic equation. In some specific conditions, the system has three steady states, the equilibrium points $E_{0}, E_{1}$ and $E_{2}$ (the system always has the equilibrium point $E_{0}$ ). The analysis of the stability of these equilibrium points was performed and eventually the existence of a Hopf bifurcation was proved for $E_{0}$ as the delay $\tau_{1}$ is varied. The equilibrium points $E_{2}$ are proved to be unstable.

Special attention was assigned to the estimation of all model parameters. Whenever possible, data collected from human subjects and published in the literature were employed. Some of the physiological processes were modeled using feedback functions, all being monotone decreasing Hill functions. Since the model has several steady states, some of them could be interpreted as corresponding to situations naturally occurring during the course of the disease. In the analysis of the model, the rate of asymmetric division and the rate of symmetric division were treated as being of most importance. Both rate values are beyond 1 as is also their sum. Theoretically, using this model, the disease evolution could be predicted if the parameters of the system were estimated, presented good accuracy or could even be tuned. This is particularly true for initial values close to one of the first two equilibrium points. This observation can make the analysis more useful and important.

Existence of periodic evolutions given by limit cycles is also an important feature highlighted by this model.

From the analysis of the model one can notice that the duration of the cell cycles $\tau_{1}, \tau_{3}$ are most influential for the behavior of other variables of the system.

Numerical simulations illustrate the effects of the $\mathrm{T}$ cell response onto the dynamics of leukemia.

There is still much work to be done in the direction of describing the natural leukemic cells dynamics and behavior. The model could help establishing how immunotherapy is to be best integrated into the standard schemes of chemotherapy helping to eliminate minimal residual disease. Our mathematical model can be used also to imagine a particular situation and solve it. The model could find applications in other fields such as antitumor-vaccine production and therapeutic management of leukemias. Also our results can be manipulated, by changing some of the parameters necessary to achieve the conditions required in the 10th Theorem, in order to obtain a Hopf bifurcation for the equilibrium point $E_{0}$. Theoretically, cyclical extension of the immune response could be obtained if the micro environmental factors, that make possible the augmentation of the "autocrine" and "positive growth signal" function, could be determined.

The numerical results were in agreement with our expectations, confirming our hypothesized findings. All the numerical simulations were done using Matlab and Maple.

In our next paper we would like to introduce the distributed time delay as in [3], [24].

Acknowledgements. We thank professor Dumitru Opris for useful conversations on the topics of this paper. This work was supported by CNCS-ROMANIA Grant ID-PCE-2011-3-0198. The authors would like to express their gratitude for valuable recommendations on this manuscript from the anonymous referees.

\section{References}

[1] A. K. Abass, A. H. Lichtman, S. Pillai. Cellular and molecular immunolgy. 7th edition, Elsevier (2012).

[2] L.H. Abbott, F. Michor. Mathematical models of targeted cancer therapy. British Journal of Cancer 95 (2006), 11361141.

[3] M. Adimy, F. Crauste. Delay differential equations and autonomous oscillations in Hematopoietic stem cell dynamics modeling. Math. Model. Nat. Phenom., (2012), 7(6), 1-22.

[4] M. Adimy, F. Crauste, A. Halanay, M. Neamţu, D. Opriş. Stability of limit cycles in a pluripotent stem cell dynamics model. Chaos, Solitons\&Fractals (2006), 27(4), 1091-1107.

[5] M. Adimy, F. Crauste, S. Ruan. A mathematical study of the hematopoiesis process with application to chronic myelogenous leukemia. SIAM J. Appl. Math. (2005), 65(4), 1328-1352. 
[6] J. Beckman, S. Scheitza, P. Wernet, J. Fischer, B. Giebel. Asymmetric cell division within the human hematopoietic stem and progenitor cell compartment: identification of asymetrically segregating proteins. Blood (2007), No. 12, 109, 5494-5501.

[7] R. Bellman, K. L. Cooke. Differential-Difference equations. Academic Press New York, (1963).

[8] E. Beretta, Y. Kuang. Geometric stability switch criteria in delay differential dystems with delay-dependent darameters. SIAM J. Math. Anal. (2002), 33(5), 1144-1165.

[9] E. Burger. On stability of certain economic systems. Econometrica (1956), 24, 488-493.

[10] C. Colijn, M.C. Mackey. A mathematical model of hematopoiesis I-Periodic chronic myelogenous leukemia. J. Theor. Biology (2005), 237, 117-132.

[11] K. Cooke, Z. Grossman. Discrete Delay, Distribution delay and stability switches. J. Math. Anal. Appl. (1982), 86, 592-627.

[12] K. Cooke, P.van den Driessche. On zeros of some transcendental equations. Funkcialaj Ekvacioj (1986), 29, 77-90.

[13] L.E. El'sgol'ts, S.B. Norkin. Introduction to the theory of differential equations with deviating arguments. (in Russian). Nauka, Moscow, 1971.

[14] A. Fridman. Cancer as multifaceted disease. Math. Model. Nat. Phenom (2012), 7, No.1, 3-28.

[15] A. Halanay. Periodic solutions in mathematical models for the treatment of chronic myelogenous leukemia. Math. Model. Nat. Phenom (2012), 7, No.1, 235-244.

[16] J. Hale. Theory of functional differential equations. Springer, New York, 1977.

[17] P. Kim, P.Lee, D. Levy. Dynamics and potential impact of the immune response to chronic myelogenous leukemia. PLoS Comput.Biol. (2008), 4(6):e 1000095.

[18] P. Kim, P.Lee, D. Levy. A theory of immunodominance and adaptive regulation,Bull. Math. Biol. (2010), DOI $10.1007 / \mathrm{s} 11538-010-9585-5$.

[19] M.C. Mackey, C. Ou, L. Pujo-Menjouet, J. Wu. Periodic oscillations of blood cell population in chronic myelogenous leukemia. SIAM J. Math. Anal. (2006), 38, 166-187.

[20] A. Marciniak-Czochra, T. Stiehl, W. Wagner. Modeling of replicative senescence in hematopoietic development. Aging (2009), 1(8), 723-732.

[21] F. Michor, T. Hughes, Y. Iwasa, S. Branford, N.P. Shah, C. Sawyers, M. Novak. Dynamics of chronic myeloid leukemia. Nature (2005), 435, 1267-1270.

[22] H. Moore, N.K. Li. A mathematical model for chronic myelogenous leukemia (CML) and T-cell interaction. J. Theor. Biol. (2004), 227, 513-523.

[23] S. I. Niculescu, P. S. Kim, K. Gu, P. Lee, D. Levy. Stability crossing boundaries of delay systems modeling immune dynamics in leukemia. Discrete and Continuous Dynamical Systems (2010), Series B Volume 13, No. 1, pp. 129-156.

[24] H. Ozbay, C. Bonnet, H. Benjelloun, J. Clairambault. Stability analysis of cell dynamcis in leukemia. Math. Model. Nat. Phenom. (2012), Volume 7, No. 1, 203-234.

[25] R. Radulescu, D. Candea, A. Halanay. Stability and bifurcation in a model for the dynamics of stem-like cells in leukemia under treatment. American Institute of Physics Proceedings (2012), 1493, 758-763.

[26] T. Reya.Regulation of hematopoietic stem cell self-Renewal. Recent Progress in Hormone Research (2003), 58, $283-295$.

[27] T. Stiehl, A. Marciniak-Czochra. Mathematical modeling of leukemogenesis and cancer stem cell dynamics. Math. Model. Nat. Phenom. (2012), Vol. 7, No. 1, 166-202.

[28] C. Tomasetti, D. Levi. Role of symmetric and asymmetric division of stem cells in developing drug resistance. PNAS (2010), Vol. 17 , No. 39, 16766-16771.

[29] J. Zajac, L. E. Harrington. Immune response to viruses: antibody-mediated immunity. University of Alabama at Birmingham, Birmingham, AL, USA, Elsevier Ltd, 2008. 